\title{
IL6ST-induced muscarinic receptor opens goblet cell associated antigen passages to suppress alcoholic liver disease
}

Michael Karin ( $\sim$ karinoffice@ucsd.edu )

University of California, San Diego https://orcid.org/0000-0002-2758-6473

Cristina Llorente

University of California San Diego https://orcid.org/0000-0001-8135-9186

Ryan Bruellman

Université Catholique de Louvain

Noemí Cabré

University of California, San Diego

Rocío Brea

University of California, San Diego

Nuria Pell

University of California, San Diego

Luca Maccioni

Université Catholique de Louvain

Ameera Alghafri

University of California, San Diego

Rongrong Zhou

Xiangya Hospital, Central South University, and Key Laboratory of Viral Hepatitis

Bei Gao

University of California, San Diego

Yi Duan

University of California, San Diego

Junlai Liu

University of California, San Diego

Koji Taniguchi

Keio University School of Medicine and Hokkaido University

Rodney Newberry

Washington University in St. Louis, School of Medicine https://orcid.org/0000-0002-4152-5191

\section{Derrick Fouts}

J. Craig Venter Institute https://orcid.org/0000-0003-4323-7668

David Brenner 
University of California, San Diego

\section{Peter Starkel}

Cliniques Universitaires St. Luc https://orcid.org/0000-0003-2938-4442

\section{Bernd Schnabl}

VA San Diego Healthcare System

\section{Biological Sciences - Article}

Keywords: alcoholic liver disease, liver damage

Posted Date: April 3rd, 2021

DOI: https://doi.org/10.21203/rs.3.rs-366644/v1

License: (9) This work is licensed under a Creative Commons Attribution 4.0 International License. Read Full License 
4 Cristina Llorente ${ }^{1}$, Ryan Bruellman ${ }^{1}$, Noemí Cabré ${ }^{1}$, Rocío Brea ${ }^{1}$, Nuria Pell ${ }^{1}$, Luca Maccioni ${ }^{2}$,

\section{IL6ST-induced muscarinic receptor opens goblet cell associated antigen passages to} suppress alcoholic liver disease

Ameera Alghafri ${ }^{1}$, Rongrong Zhou ${ }^{1,3}$, Bei Gao ${ }^{1}$, Yi Duan ${ }^{1}$, Junlai Liu ${ }^{4}$, Koji Taniguchi ${ }^{4,5,}$, Rodney D. Newberry ${ }^{7}$, Derrick E. Fouts ${ }^{8}$, David A. Brenner ${ }^{1}$, Peter Stärkel ${ }^{2,9}$, Michael Karin ${ }^{4 *}$, Bernd Schnabl $1^{1,10 *}$

${ }^{1}$ Department of Medicine, University of California San Diego, La Jolla, CA, USA $;{ }^{2}$ Institute of Experimental and Clinical Research, Laboratory of Hepato-gastroenterology, UCLouvain, Université Catholique de Louvain, Brussels, Belgium; ${ }^{3}$ Department of Infectious Diseases, Xiangya Hospital, Central South University, and Key Laboratory of Viral Hepatitis, Hunan, Changsha, China; ${ }^{4}$ Laboratory of Gene Regulation and Signal Transduction, Departments of Pharmacology and Pathology, University of California San Diego, La Jolla, CA, USA; ${ }^{5}$ Department of Microbiology and Immunology, Keio University School of Medicine, Shinjukuku, Tokyo, Japan; ${ }^{6}$ Department of Pathology, Faculty of Medicine and Graduate School of Medicine, Hokkaido University, Sapporo, Hokkaido, Japan; ${ }^{7}$ Department of Internal Medicine, Washington University School of Medicine, St. Louis, Missouri, USA; ${ }^{8}$ J. Craig Venter Institute, Rockville, MD, USA; ${ }^{9}$ Department of Hepato-gastroenterology, Cliniques Universitaires SaintLuc, Brussels, Belgium; ${ }^{10}$ Department of Medicine, VA San Diego Healthcare System, San Diego, 1 CA, USA 
24 Abbreviations: ACh, acetylcholine; ALD, alcoholic liver disease; ALT, alanine amino25 transferase; AMP, antimicrobial peptides; ANOVA, analysis of variance; APCs; antigen 26 presenting cells, AUD, alcohol use disorder; $\mathrm{BHI}$ broth, brain heart infusion broth; $\mathrm{CD}$, cluster 27 of differentiation; CFU, colony forming unit; CK18, cytokeratin 18; Cxcl, chemokine (C-X-C 28 motif); CX3CR1, C-X3-C Motif Chemokine Receptor; DC, dendritic cell; E. faecalis; 29 Enterococcus faecalis; EGFP, green fluorescent protein; FOXP3, forkhead box P3; GAP, goblet 30 cell associated antigen passage; GC, goblet cell; H\&E, hematoxylin and eosin; IEC, intestinal 31 epithelial cells; IL, interleukin; ILC, innate lymphoid cell; IL6ST, IL-6 signal transducer; LP, 32 lamina propria; $\mathrm{mAChR}$, muscarinic $\mathrm{ACh}$ receptor; $\mathrm{MHC}$, major histocompatibility class; MLN, 33 mesenteric lymph node; ORO, oil red O; OTU, operational taxonomic unit; PAM, positive 34 allosteric modulator; PAS, Periodic Acid/Schiff; PBS, phosphate-buffered saline; PCoA, principal 35 36 receptor; Treg, T regulatory cell; WT, wild type. 


\section{Summary}

48 Alcohol consumption is the seventh leading risk factor for death worldwide ${ }^{1}$ and alcoholic liver

49 disease (ALD) is the major cause of liver transplantation in the West ${ }^{2}$. Alcohol metabolites can

50 directly damage the liver, but the gut-liver axis also plays an important role in ALD pathogenesis,

51 acting through complex and obscure mechanisms. Although alcohol intake is associated with

52 increased intestinal permeability ${ }^{3}$, it is not clear how the intestinal immune system affects liver

53 disease. Paradoxically, we found that chronic alcohol use increases intestinal goblet cell (GC)

54 number and mucin production in patients and mice. This, however, comes with the expense of

55 GC associated antigen passages (GAPs) closure and defective delivery of luminal antigens and

56 bacteria to lamina propria (LP) antigen presenting cells (APCs). IL-6 is one of several cytokines

57 elevated in ALD that affects the liver and the intestine ${ }^{4}$. Although IL-6 expression correlates with

58 disease severity ${ }^{5}$, IL-6 also exerts barrier protective effects ${ }^{6}$. We now show that GAPs are

59 controlled by intestinal IL-6 signal transducer (IL6ST/gp130). Although mice that express

60 constitutively active gp130 in intestinal epithelial cells (IEC; gp130 ${ }^{A c t / I E C}$ mice) have fewer GCs

617 , they are ALD resistant due to increased GAP formation, which enhances the generation of 62 tolerogenic LP-APCs and production of IL-22 by type-3 innate lymphoid cells (ILC3s). GAP

63 opening induces an intestinal C-type regenerating islet derived-3 (Reg3) lectin-mediated

64 antibacterial defense, reducing bacterial translocation to the liver and preventing alcoholic

65 steatohepatitis. gp130 signaling exerted its protective effects via muscarinic acetylcholine (ACh)

66 receptor 4 (mAChR4), whose GC expression was induced by IL-6. Based on these findings, we

67 developed a new therapeutic approach, administering a mAChR4 positive allosteric modulator

68 (PAM) to stimulate intestinal GAP formation, thereby increasing tolerogenic LP-APCs and Reg3

69 expression, to prevent microbial translocation and protect mice from alcoholic steatohepatitis. 
70 Our results show that IL6ST signaling modulates intestinal immunity through mAChR4. GAP

71 induction by mAChR4 PAMs is a promising strategy for enhancing intestinal immune tolerance

72 and interception in ALD and other diseases linked to uncontrolled microbial translocation.

73

74

75

76

77

78

79

80

81

82

83

84

85

86

87

88

89

90

91

92 
94 Alcohol consumption is the seventh leading risk factor for death worldwide ${ }^{1}$, and ALD is the 95 major cause of liver transplantation in the West ${ }^{2}$. There is no treatment for ALD except for 96 abstinence. Chronic alcohol abuse is associated with increased intestinal permeability, dysbiosis 97 and translocation of viable bacteria, such as Enterococcus faecalis (E. faecalis), to the liver, 98 enhancing inflammation and accelerating ALD progression ${ }^{8,9}$. Intestinal immune-microbiome 99 interactions are altered during ALD ${ }^{10}$. Such findings suggest that the intestinal immune system 100 may control ALD pathogenesis. To test this hypothesis and elucidate the underlying mechanisms 101 we investigated how chronic alcohol exposure affects the intestinal immune system and bacterial 102 translocation to the liver. The findings described below are of relevance to other diseases 103 associated with loss of intestinal barrier integrity.

\section{Chronic alcohol abuse alters GC biology in mice and humans}

106 GCs secrete mucins which coat the IEC apical surface and prevent microbial adhesion and 107 translocation ${ }^{11}$. Paradoxically, chronic ethanol administration increased production of mucin-2 108 (Muc2), the most abundant intestinal mucin ${ }^{12}$, and the number of small intestine mucin secreting 109 GCs in mice (Fig. 1a-1b) and patients with alcohol use disorder (AUD) (Fig. 1c-1d). Small 110 intestinal GCs deliver luminal antigens and bacteria to LP-APCs through GAPs, thereby inducing 111 adaptive immune responses ${ }^{13,14}$. GAPs are formed immediately after mucin is secreted into the 112 intestinal lumen ${ }^{13}$. We observed that the number of GAPs, identified as dextran-filled columns 113 traversing Muc2 positive nucleated cells, was reduced following chronic ethanol feeding (Fig. 1e114 1f). Small intestinal GAPs are dynamic, and they open in response to ACh, which engages 115 muscarinic mAChR4 on GCs ${ }^{15,16}$. The observed reduction in GAP formation after ethanol 
exposure could be related to reduced expression of Chrm4, the gene for mAChR4 (Fig. $1 \mathrm{~g}$ ). When we gavaged mice with enhanced green fluorescent protein (EGFP)-E. faecalis, we found colocalization of bacteria with Muc2 inside GCs (Fig. 1h) and with dextran-filled GAP columns (Fig. 1i), suggesting that bacteria are sampled by GCs. These results indicate that chronic alcohol use increases protective mucin production, which paradoxically comes with the expense of GAP closure.

\section{IL6ST-mediated induction of GAPs prevents ethanol-induced liver disease}

IL-6 is one of several cytokines elevated in ALD that affects the liver and the intestine ${ }^{4}$. Although IL-6 correlates with liver disease severity ${ }^{5}$, it also exerts important barrier protective effects ${ }^{6}$. Of note, IL-6 treatment induced mAChR4 expression in small intestinal organoids (Fig. 2a-2c). Previously, we showed that IL-6 signaling promotes intestinal stem cell survival and proliferation by activating STAT3 and YAP and that $g p 130^{\text {Act/IEC }}$ mice are resistant to mucosal erosion, despite having fewer GCs than wild type (WT) mice ${ }^{7}$. Remarkably, gp $130^{A c t / I E C}$ mice were protected from ethanol-induced liver injury compared with WT littermates, as indicated by lower plasma alanine amino-transferase (ALT) (Fig. 2d) and reduced hepatic steatosis (Fig. 2e2f) and inflammation (Fig. 2g-2h), while having fewer GCs and lower Muc2 expression in the small intestine (Fig. 2i-2j). Activation of gp130 in IEC did not affect food intake or intestinal ethanol absorption (Extended Data Fig. 1a-1b). In accordance with the in vitro experiments, gp130 ${ }^{A c t / I E C}$ mice showed more GAPs in the small intestine (Fig. 2k-21), expressed higher amounts of Chrm4 mRNA (Fig. 2m) and mAChR4 protein in isolated GCs (Fig. 2n-2o and Extended Data Fig. 1c-1d). mAChR4 is expressed in IEC, GC, some LP immune cells and the enteric nervous system in the small intestine. mACR4 in GC was distributed above the nuclei and subjacent to the 
secretory granules (Extended Data Fig. 1e). Ethanol-fed gp130 $0^{A c t / I E C}$ mice were protected from intestinal bacterial overgrowth and showed a different fecal microbiota composition than WT littermates (Fig. 2p-2q). These results indicate that IL-6-gp130 regulation of intestinal GAPs impacts the development of ethanol-induced liver injury.

\section{gp130 ${ }^{A c t / I E C}$ mice show elevated tolerogenic APCs and antimicrobial defense}

To study the consequences of GAP modulation, we characterized different subsets of CD11c $\mathrm{c}^{+}$, major histocompatibility class (MHC)II ${ }^{+}$LP-APCs ${ }^{17}$. Chronic ethanol-feeding in WT mice did not affect frequencies or numbers of $\mathrm{CD} 11 \mathrm{c}^{+}, \mathrm{MHCII}^{+} \mathrm{APCs}$ (Extended Data Fig. 2a-2c), but it

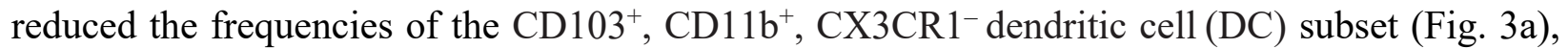

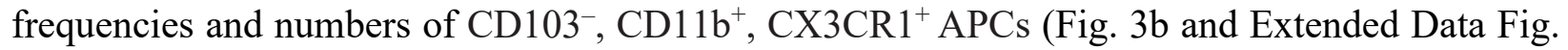
2d), numbers of IL-23 expressing APC subsets (Fig. 3c), and IL-10 expressing APC subsets (Extended Data Fig. 2e). Correspondingly, ethanol decreased frequencies of ILC3s (CD3-, ROR $\gamma \mathrm{t}^{+}$) and IL-22 expressing ILC3s (Fig. 3d-3f).

Alcohol-induced suppression of the intestinal immune system was restored in $g p 130^{A c t / I E C}$ mice,

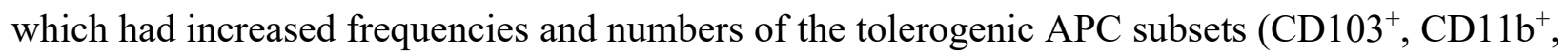

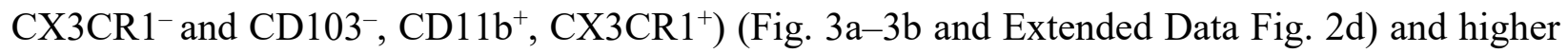
numbers of IL-23 and IL-10 expressing APC subsets (Fig. 3c and Extended Data Fig. 2e). Frequencies of ILC3s $\left(\mathrm{CD}^{-}, \mathrm{ROR}_{\gamma} \mathrm{t}^{+}\right)$were also increased in ethanol-fed gp130 1 Act/IEC mice along with the number of IL-22 expressing ILC3s (Fig. 3d-3f). As a consequence, the Reg3g and Reg3b antimicrobial peptides were upregulated in $g p 130^{A c t / I E C}$ mice after ethanol administration (Fig. $3 \mathrm{~g}-$ 3i). As we have reported, increased Reg3 lectins might not only contribute to protection from bacterial overgrowth (Fig. $2 p$ ) but can also inhibit bacterial translocation ${ }^{18}$. Indeed, less viable 
162

163

164

165

166

167

168

169

170

171

172

173

174

175

176

177

178

179

180

181

182

183

184

bacteria translocated from the intestine to mesenteric lymph nodes (MLNs) and liver of ethanolfed $g p 130^{A c t / I E C}$ mice relative to WT mice (Fig. $3 \mathrm{j}-3 \mathrm{k}$ ). Consistent with the increase in tolerogenic APC subsets, frequencies and numbers of $\mathrm{T}$ regulatory cells (Tregs) characterized as $\mathrm{CD}^{+}$, $\mathrm{CD}^{2} 5^{+}, \mathrm{FOXP}^{+}$and levels of intestinal IL-10 were elevated in $g p 130^{A c t / I E C}$ mice after ethanol treatment (Extended Data Fig. 3a-3e). These results indicate that a specific tolerogenic APCmediated intestinal adaptive immune response and antibacterial defenses might contribute to protection against ethanol-induced liver disease in mice.

\section{gp130 $0^{A c t / I E C}$ mice lose protection from ethanol-induced liver disease on mAChR4 antagonism}

To determine whether gp130 exerts its protective effects via mAChR4-controlled GAP opening, mice were treated with the mAChR4 antagonist tropicamide. Tropicamide dissolved in the diet during the last 29 days of chronic ethanol feeding inhibited intestinal GAP formation (Fig. 31-3m and Extended Data Fig. 4a) and made gp130 $0^{A c t / I E C}$ mice lose protection from liver disease (Fig. 4n and Extended Data Fig. 4b), steatosis (Fig. 3o-3p and Extended Data Fig. 4c) and inflammation (Fig. 4q, Extended Data Fig. 4d-4e). Despite a tendency, tropicamide did not worsen the disease in WT mice, probably because ethanol already inhibited GAP formation in these mice (Fig. 1e1g, Extended Data Fig. 4a). Tropicamide did not affect food intake or intestinal ethanol absorption (Extended Data Fig. 1a-1b). Tropicamide abrogated the increase in GAP-associated CD103+, $\mathrm{CD}_{11} \mathrm{~b}^{+}, \mathrm{CX} 3 \mathrm{CR} 1^{-} \mathrm{DCs}$ and $\mathrm{CD} 103^{-}, \mathrm{CD} 11 \mathrm{~b}^{+}, \mathrm{CX} 3 \mathrm{CR} 1^{+} \mathrm{APCs}, \mathrm{ILC} 3 \mathrm{~s}$ and Tregs in ethanol-fed gp130 $0^{A c t / I E C}$ mice (Fig. 3a-3b, 3d-3f, Extended Data Fig. 2d and Extended Data Fig. 3a-3c). Tropicamide treatment also reversed Reg3b and Reg3g protein induction in ethanol-fed gp $130^{A c t / I E C}$ mice (Fig. 3r-3t), and significantly increased bacterial translocation to MLN and liver in ethanol-fed gp130 $0^{A c t / I E C}$ mice (Fig. 3j-3k). Consistent with these results which suggest that IL- 
1856 induces mAChR4 whose activity is needed for IL-22 production and subsequent Reg3 induction, 186 incubation of small intestinal organoids with IL-6 resulted in Chrm4 but not Reg3g or Reg3b 187 mRNA induction, whereas IL-22 induced Reg3g and Reg3b but not Chrm 4 mRNA (Extended Data 188 Fig. $4 \mathrm{f}-4 \mathrm{~h})$. These data demonstrate that intestinal gp130 activation exerts its liver protective 189 effects via mAChR4-induced GAP formation.

\section{Pharmacological manipulation of GAPs alleviates ethanol-induced liver injury}

Based on these findings, we developed a new therapeutic approach, administering a mAChR4 PAM, VU0467154, to stimulate intestinal GAP formation. WT mice fed an ethanol containing diet for 10 weeks, were treated with VU0467154 during the last 29 days. VU0467154 has no 195 gastrointestinal motility side effects and excellent oral bioavailability ${ }^{19-21}$. VU0467154 treatment abrogated ethanol-induced GAP closure (Fig. 4a-4b), and protected mice from ethanol-induced liver injury (Fig. 4c-4d), steatosis (Fig. 4e-4f) and inflammation (Fig. 4g). mAChR4 PAM treatment did not affect intestinal ethanol absorption, as pair-fed mice had similar blood alcohol levels (Extended Data Fig. 5a-5b). Frequencies of LP-APCs $\left(\mathrm{CD} 11 \mathrm{c}^{+}, \mathrm{MHCII}^{+}\right)$and $\mathrm{CD}_{103}^{+}$ CD11b ${ }^{+}$DC populations in ethanol fed mice were increased by VU0467154 treatment (Fig. 4h4k), which also induced intestinal Reg3g, Reg3b and IL-10 expression (Fig. 4l-4o) and prevented ethanol-mediated bacterial translocation to MLNs and liver (Fig. 4p-4q). Our results indicate that pharmacological manipulation of $\mathrm{mAChR} 4$ reduced ethanol-induced steatohepatitis.

\section{Discussion}

206 This study reveals a fine balance between physical barrier function and a protective intestinal

207 immune response. Chronic alcohol increases intestinal mucin production, but this comes at the 
expense of GAP closing and improper training of intestinal immunity. LP-APCs adjacent to GAPs induce specific protective immune responses that control intestinal homeostasis $13,15,16,22,23$. Regenerative responses are important after intestinal injury or microbial infection to maintain barrier integrity and prevent translocation of intestinal bacteria ${ }^{24,25}$. gp130 signaling stimulates regeneration and regulates differentiation of the gastrointestinal barrier through YAP and Notch activation ${ }^{7,26-29}$. Only very recently it was determined that inhibition of a dynamic neuroimmune circuit by activation of VIP receptor type 2 (VIPR2) in the intestine triggers IL-22 secretion by ILC3s and increases Reg3g mRNA in ileum ${ }^{30}$. Our findings add to the complex neuronal regulation of antibacterial defenses. Intestinal IL6ST/gp130 controls cholinergic signaling by upregulating mAChR4 on GCs to induce GAP formation (Extended Data Fig. 6). Therefore, IL6ST/gp130 signaling enhances the GC response to ACh released by GC-innervating parasympathetic neurons and possibly other cellular sources.

Ethanol therefore causes liver disease not only via direct toxic effects on liver cells, but also by suppressing the intestinal immune system and allowing enteric bacteria to translocate to the liver, underscoring the importance of the gut-liver axis for ALD pathogenesis. Consistent with this finding, pharmacological manipulation of mAChR4 with a PAM to induce small intestinal GAPs was associated with modulation of APCs, induction of Reg3 lectins, prevention of bacterial translocation and amelioration of ALD. Of note, whereas IL-6 induces mAChR4, IL-22 induces Reg3 lectins, suggesting that two cytokines act hierarchically and through different protective mechanisms, although both of them stimulate mucosal regeneration ${ }^{31}$. Interestingly, mAChR4 gene and protein expression are modulated in specific brain regions of AUD patients. The number of muscarinic receptors increases in several brain regions in the absence of alcohol during withdrawal ${ }^{32}$. Systemic administration of VU0467154 reduced ethanol seeking and consumption 
231 in a rodent model ${ }^{33}$. We did not examine alcohol consumption in our mice, as they were pair-fed 232 and received the same amount of ethanol-containing diet. Nevertheless, pharmacological 233 manipulation of $\mathrm{mAChR} 4$ could be a potential approach for preventing not only ALD progression, 234 but also for treating AUD. Targeted delivery of intestinal gp130 agonists to GCs and mAChR4 235 PAMs might be developed as a therapeutic agent for alcohol-related liver disease.

236

237

238

239

240

241

242

243

244

245

246

247

248

249

250

251

252 


\section{References}

254

255

256

257

258

259

260

261

262

263

264

265

266

267

268

269

270

271

272

273

274

275

276

277

278

279

280

281

282

283

284

285

286

287

288

289

290

291

292

293

294

295

296
1 Collaborators, G. B. D. A. Alcohol use and burden for 195 countries and territories, 19902016: a systematic analysis for the Global Burden of Disease Study 2016. Lancet 392, 10151035, doi:10.1016/S0140-6736(18)31310-2 (2018).

2 Lee, B. P., Vittinghoff, E., Dodge, J. L., Cullaro, G. \& Terrault, N. A. National Trends and Long-term Outcomes of Liver Transplant for Alcohol-Associated Liver Disease in the United States. JAMA Intern Med 179, 340-348, doi:10.1001/jamainternmed.2018.6536 2720757 [pii] (2019).

3 Lang, S. \& Schnabl, B. Microbiota and Fatty Liver Disease-the Known, the Unknown, and the Future. Cell Host Microbe 28, 233-244, doi:10.1016/j.chom.2020.07.007 (2020).

4 Hong, F. et al. Elevated interleukin-6 during ethanol consumption acts as a potential endogenous protective cytokine against ethanol-induced apoptosis in the liver: involvement of induction of Bcl-2 and Bcl-x(L) proteins. Oncogene 21, 32-43, doi:10.1038/sj.onc.1205016 (2002).

5 Sheron, N., Bird, G., Goka, J., Alexander, G. \& Williams, R. Elevated plasma interleukin-6 and increased severity and mortality in alcoholic hepatitis. Clin Exp Immunol 84, 449-453 (1991).

6 Kuhn, K. A. et al. Bacteroidales recruit IL-6-producing intraepithelial lymphocytes in the colon to promote barrier integrity. Mucosal Immunol 11, 357-368, doi:10.1038/mi.2017.55 (2018).

7 Taniguchi, K. et al. A gp130-Src-YAP module links inflammation to epithelial regeneration. Nature 519, 57-62, doi:10.1038/nature14228 (2015).

8 Llorente, C. et al. Gastric acid suppression promotes alcoholic liver disease by inducing overgrowth of intestinal Enterococcus. Nat Commun 8, 837, doi:10.1038/s41467-017-00796$\mathrm{x}(2017)$.

9 Duan, Y. et al. Bacteriophage targeting of gut bacterium attenuates alcoholic liver disease. Nature 575, 505-511, doi:10.1038/s41586-019-1742-x (2019).

10 Bruellman, R. \& Llorente, C. A Perspective Of Intestinal Immune-Microbiome Interactions In Alcohol-Associated Liver Disease. Int J Biol Sci 17, 307-327, doi:10.7150/ijbs.53589 (2021).

11 Velcich, A. et al. Colorectal cancer in mice genetically deficient in the mucin Muc2. Science 295, 1726-1729, doi:10.1126/science.1069094 (2002).

12 Hartmann, P. et al. Deficiency of intestinal mucin-2 ameliorates experimental alcoholic liver disease in mice. Hepatology 58, 108-119, doi:10.1002/hep.26321 (2013).

13 McDole, J. R. et al. Goblet cells deliver luminal antigen to CD103+ dendritic cells in the small intestine. Nature 483, 345-349, doi:10.1038/nature10863 (2012).

14 Kulkarni, D. H. et al. Goblet cell associated antigen passages support the induction and maintenance of oral tolerance. Mucosal Immunol 13, 271-282, doi:10.1038/s41385-019-02407 (2020).

15 Knoop, K. A., McDonald, K. G., McCrate, S., McDole, J. R. \& Newberry, R. D. Microbial sensing by goblet cells controls immune surveillance of luminal antigens in the colon. Mucosal Immunol 8, 198-210, doi:10.1038/mi.2014.58 (2015).

16 Knoop, K. A. et al. Antibiotics promote the sampling of luminal antigens and bacteria via colonic goblet cell associated antigen passages. Gut Microbes 8, 400-411, doi:10.1080/19490976.2017.1299846 (2017). 
17 Denning, T. L. et al. Functional specializations of intestinal dendritic cell and macrophage subsets that control Th17 and regulatory $\mathrm{T}$ cell responses are dependent on the T cell/APC ratio, source of mouse strain, and regional localization. J Immunol 187, 733-747, doi:10.4049/jimmunol.1002701 (2011).

18 Wang, L. et al. Intestinal REG3 Lectins Protect against Alcoholic Steatohepatitis by Reducing Mucosa-Associated Microbiota and Preventing Bacterial Translocation. Cell Host Microbe 19, 227-239, doi:10.1016/j.chom.2016.01.003 (2016).

19 Bubser, M. et al. Selective activation of M4 muscarinic acetylcholine receptors reverses MK801 -induced behavioral impairments and enhances associative learning in rodents. ACS Chem Neurosci 5, 920-942, doi:10.1021/cn500128b (2014).

20 Pancani, T. et al. Allosteric activation of M4 muscarinic receptors improve behavioral and physiological alterations in early symptomatic YAC128 mice. Proc Natl Acad Sci U S A 112, 14078-14083, doi:10.1073/pnas.1512812112 (2015).

21 Gould, R. W. et al. Cognitive enhancement and antipsychotic-like activity following repeated dosing with the selective M4 PAM VU0467154. Neuropharmacology 128, 492-502, doi:10.1016/j.neuropharm.2017.07.013 (2018).

22 Knoop, K. A., McDonald, K. G., Kulkarni, D. H. \& Newberry, R. D. Antibiotics promote inflammation through the translocation of native commensal colonic bacteria. Gut $\mathbf{6 5}, 1100$ 1109, doi:10.1136/gutjnl-2014-309059 (2016).

23 Kulkarni, D. H. et al. Goblet cell associated antigen passages are inhibited during Salmonella typhimurium infection to prevent pathogen dissemination and limit responses to dietary antigens. Mucosal Immunol 11, 1103-1113, doi:10.1038/s41385-018-0007-6 (2018).

24 Medzhitov, R. Origin and physiological roles of inflammation. Nature 454, 428-435, doi:10.1038/nature07201 (2008).

25 Ben-Neriah, Y. \& Karin, M. Inflammation meets cancer, with NF-kappaB as the matchmaker. Nature immunology 12, 715-723, doi:10.1038/ni.2060 (2011).

26 Gregorieff, A., Liu, Y., Inanlou, M. R., Khomchuk, Y. \& Wrana, J. L. Yap-dependent reprogramming of Lgr5(+) stem cells drives intestinal regeneration and cancer. Nature 526, 715-718, doi:10.1038/nature15382 (2015).

$27 \mathrm{Yu}$, S. et al. Paneth Cell Multipotency Induced by Notch Activation following Injury. Cell Stem Cell 23, 46-59 e45, doi:10.1016/j.stem.2018.05.002 (2018).

28 Todoric, J. et al. Fructose stimulated de novo lipogenesis is promoted by inflammation. Nat Metab 2, 1034-1045, doi:10.1038/s42255-020-0261-2 (2020).

29 Romera-Hernandez, M. et al. Yap1-Driven Intestinal Repair Is Controlled by Group 3 Innate Lymphoid Cells. Cell Rep 30, 37-45 e33, doi:10.1016/j.celrep.2019.11.115 (2020).

30 Talbot, J. et al. Feeding-dependent VIP neuron-ILC3 circuit regulates the intestinal barrier. Nature 579, 575-580, doi:10.1038/s41586-020-2039-9 (2020).

31 Lindemans, C. A. et al. Interleukin-22 promotes intestinal-stem-cell-mediated epithelial regeneration. Nature 528, 560-564, doi:10.1038/nature16460 (2015).

32 Nordberg, A. \& Wahlström, G. Cholinergic mechanisms in physical dependence on barbiturates, ethanol and benzodiazepines. Journal of Neural Transmission / General Section JNT 88, 199-221, doi:10.1007/BF01244733 (1992).

33 Walker, L. C. et al. Acetylcholine Muscarinic M4 Receptors as a Therapeutic Target for Alcohol Use Disorder: Converging Evidence From Humans and Rodents. Biol Psychiatry 88, 898-909, doi:10.1016/j.biopsych.2020.02.019 (2020). 
Figure 1

a

Muc2

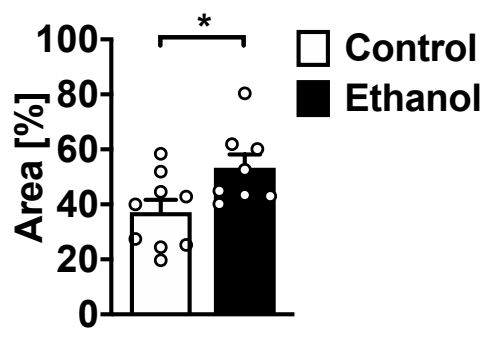

C

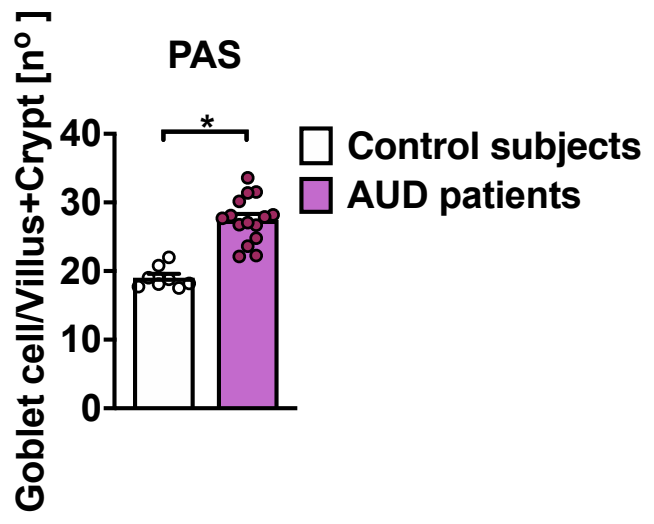

e

GAPs

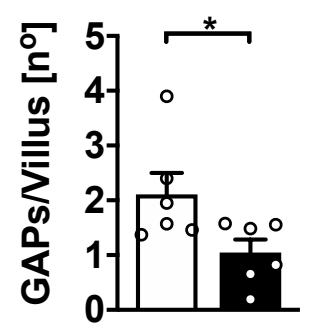

f b

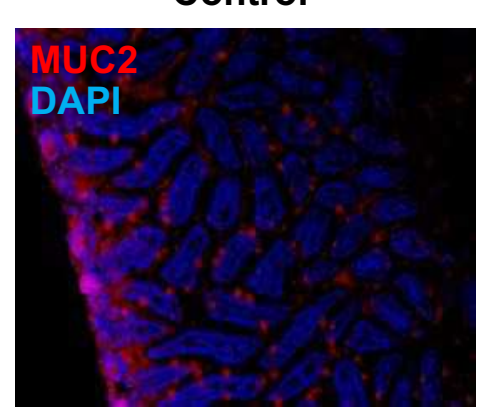

d
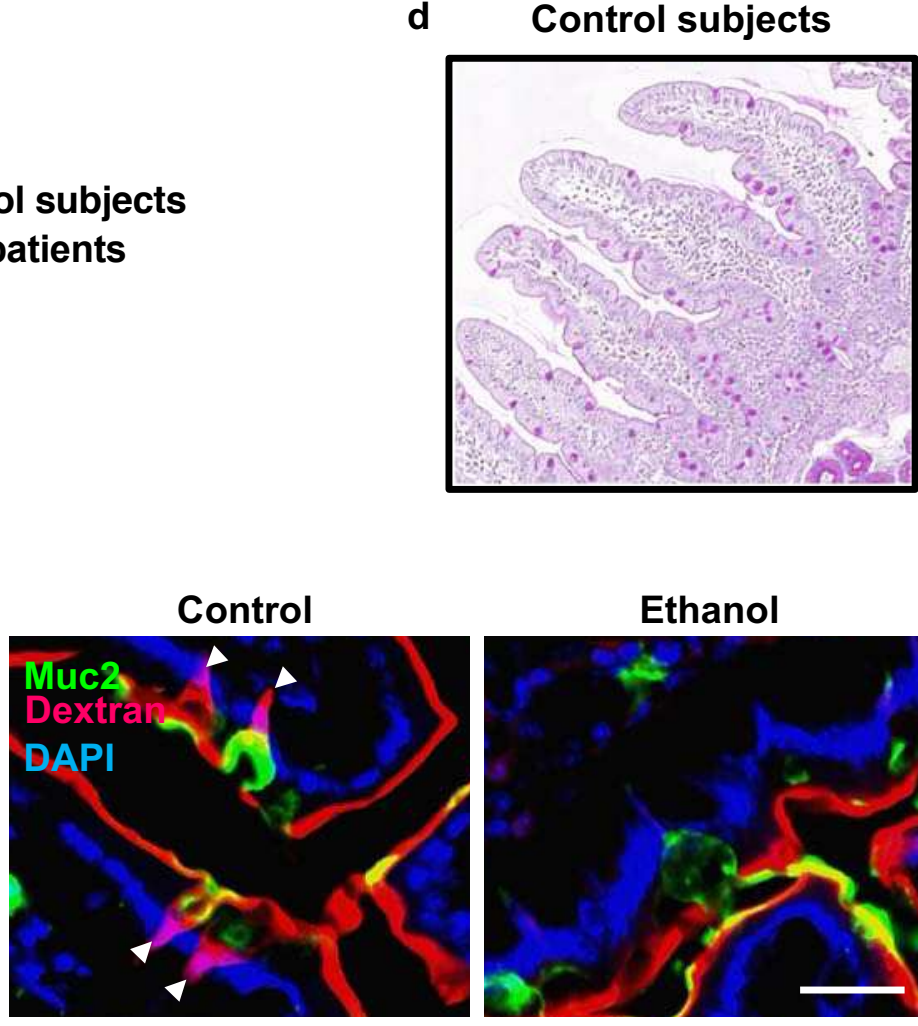

\section{Ethanol}

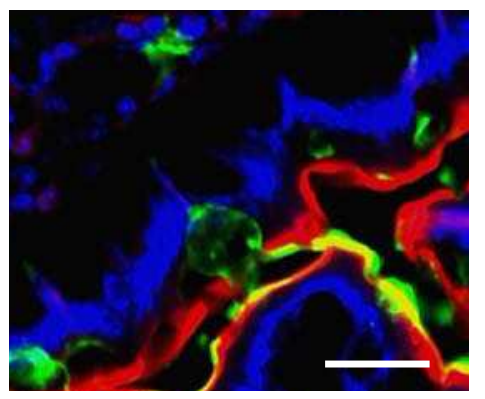

Ethanol

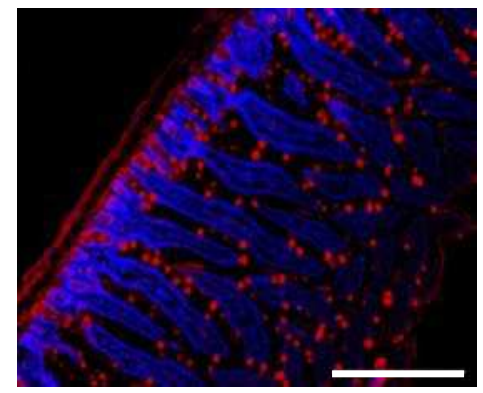

AUD patients

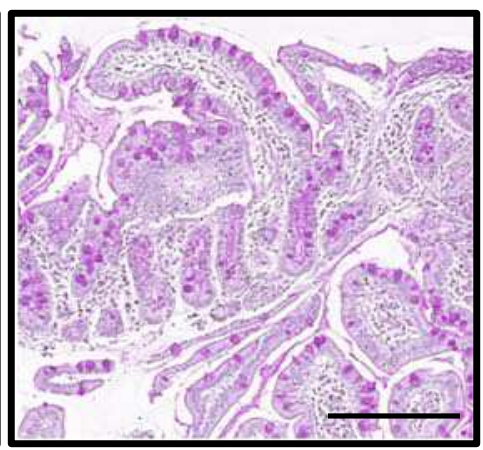

h

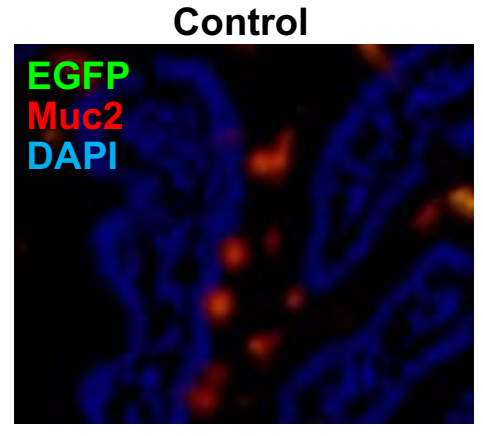

Ethanol

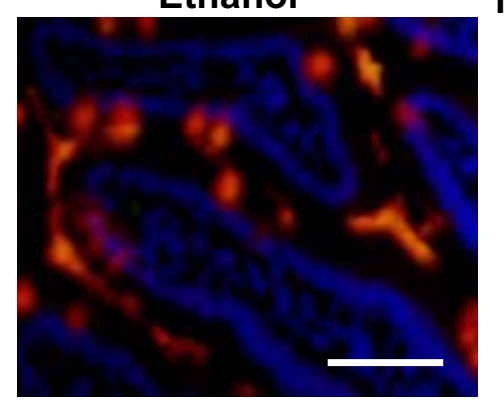

\section{Control}

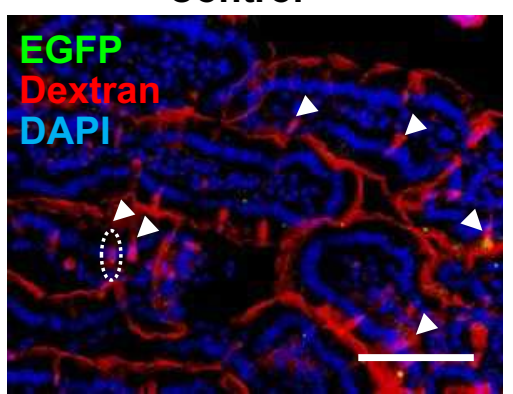

g

Chrm4

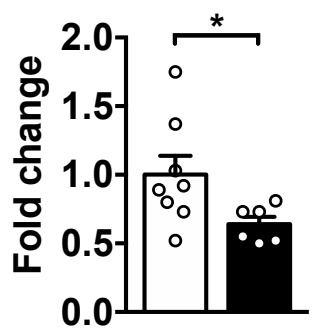

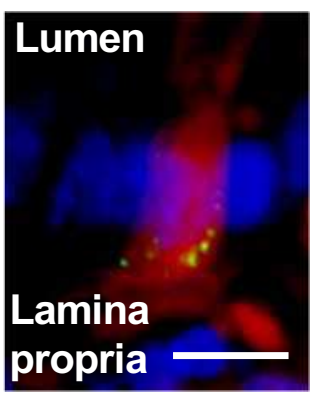


344 Figure 1. Chronic ethanol alters proximal intestinal GC in mice and humans. (a-b and e-i)

345 C57BL/6 WT mice were fed control $(n=6-9)$ or ethanol containing Lieber DeCarli diets $(n=6-8)$

346 for 10 weeks. (a) Percentage of Muc2 positive stained area. (b) Representative sections of Muc2

347 (red) showing GCs and DAPI (blue) immunofluorescence staining. Scale bar $=250 \mu \mathrm{m}$. (c, d)

348 Periodic Acid/Schiff (PAS) staining of paraffin-embedded small intestinal sections of duodenal

349 biopsies from non-alcoholic controls $(n=8)$ and patients with AUD $(n=15)$. (c) Positive cells were

350 enumerated in each villus and crypt. (d) Representative PAS-stained sections showing increased

351 number of GCs in the duodenum of AUD patients as compared with controls. Scale bar $=100 \mu \mathrm{m}$.

352 (e) To study GC GAP formation, a $2 \mathrm{~cm}$ loop in the small intestine was injected with

353 tetramethylrhodamine (TMR) dextran and GAPs were counted as dextran-filled columns

354 traversing the nucleated epithelium and positive for Muc2 staining. Number of GAPs per villus

355 was quantified. (f) Representative sections stained with TMR-dextran (red) showing GAPs, Muc2

356 antibody (green) showing GCs and DAPI (blue) marking nuclei. Scale bar $=25 \mu \mathrm{m}$. (g) Chrm4

357 mRNA was quantified by qPCR. (h) E. faecalis genetically modified with an EGFP vector (5 x

$35810^{9}$ CFUs) were gavaged $9 \mathrm{~h}$ and $1 \mathrm{~h}$ before euthanasia $(\mathrm{n}=6)$. Representative sections stained with

359 Muc2 antibody (red), EGFP (green) and DAPI (blue). Scale bar $=50 \mu \mathrm{m}$. (i) EGFP-E. faecalis (5

$360 \times 10^{9}$ CFUs) were gavaged at 3 and $0.5 \mathrm{~h}$ before euthanasia $(\mathrm{n}=6)$. Representative section of EGFP 361 (green), TMR-dextran (red) and DAPI (blue) stained intestinal sections. Left panel: scale bar = $362100 \mu \mathrm{m}$, and right panel (amplification of the white discontinuous oval): scale bar $=12 \mu \mathrm{m}$. (f and 363 i) Arrowheads indicate GAPs. $P$ value was determined by two-sided unpaired Student $t$ test or 364 Mann-Whitney U-statistic test. Results are expressed as mean \pm s.e.m. $* P<0.05$. 
Figure 2

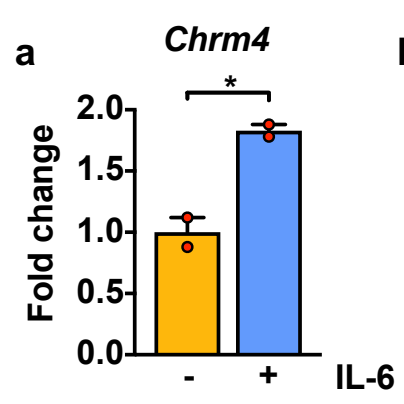

ALT

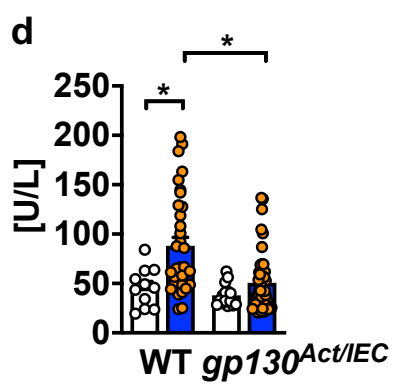

Cxc/1

9
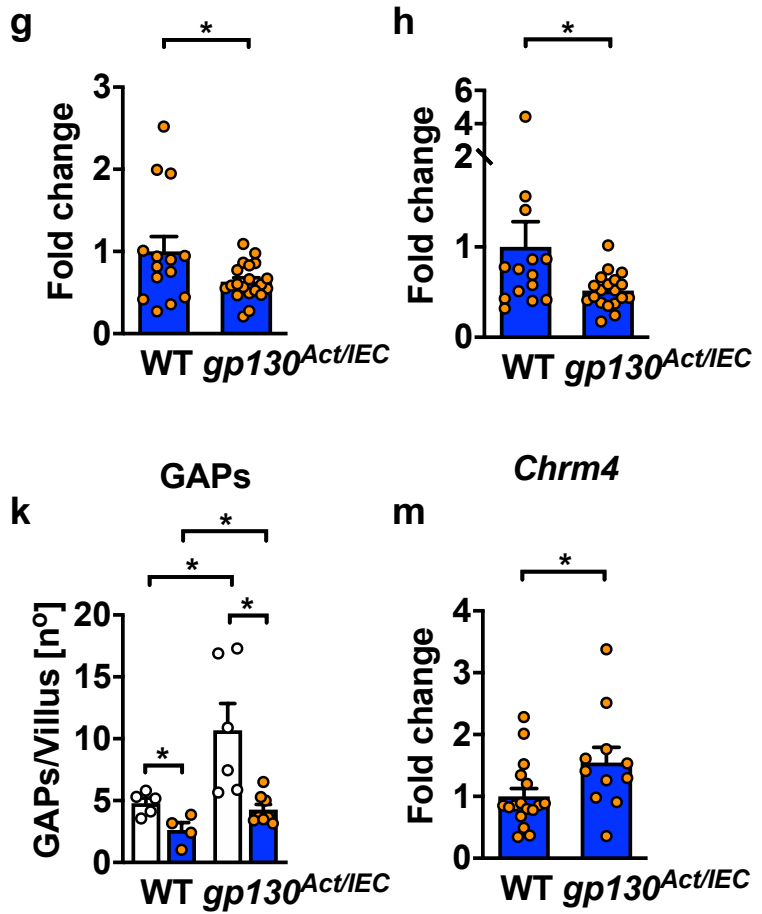

Chrm4

m
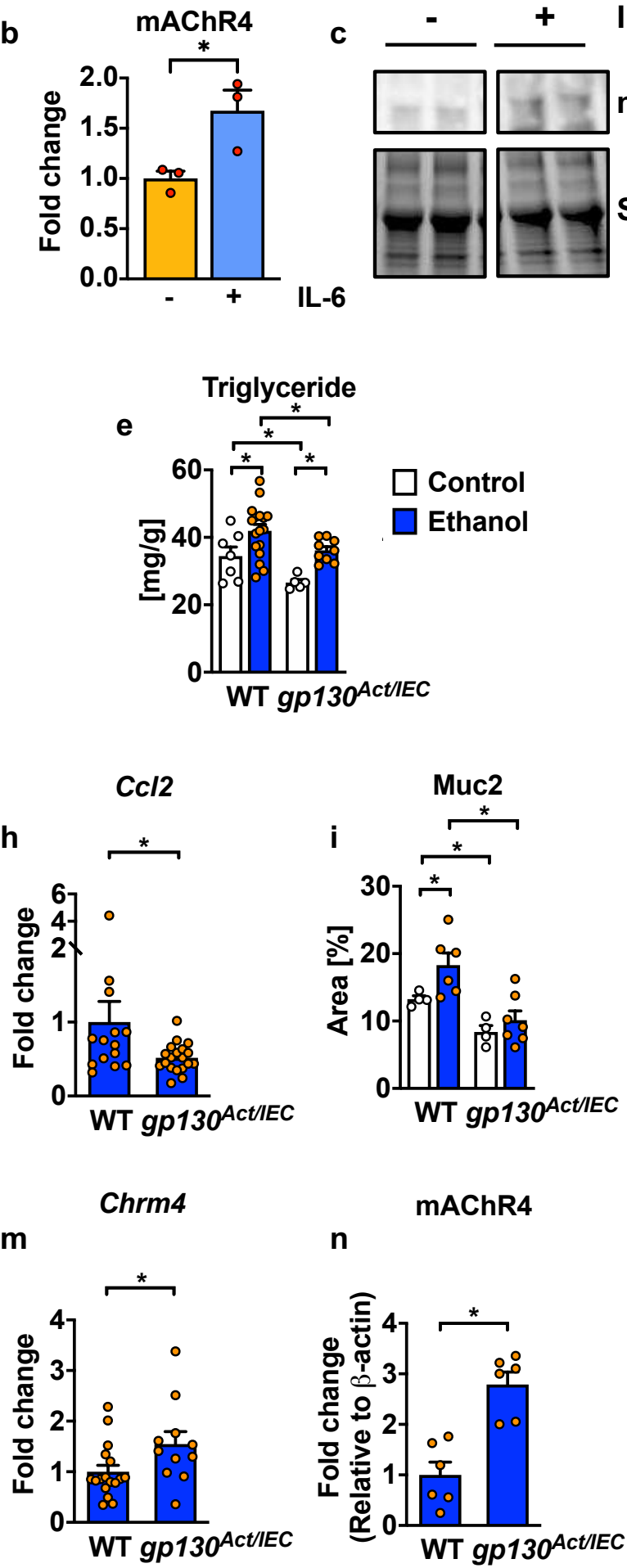

Cc/2

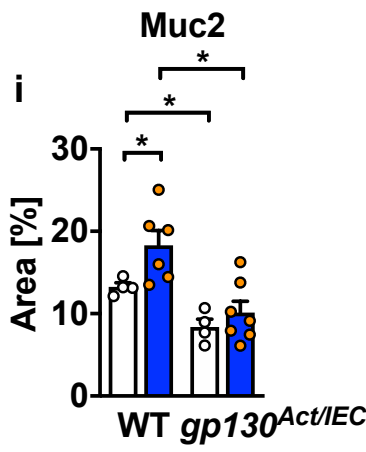

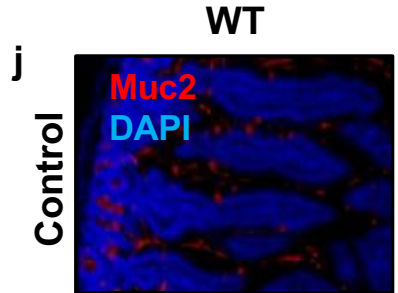

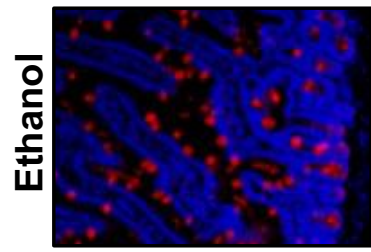

WT
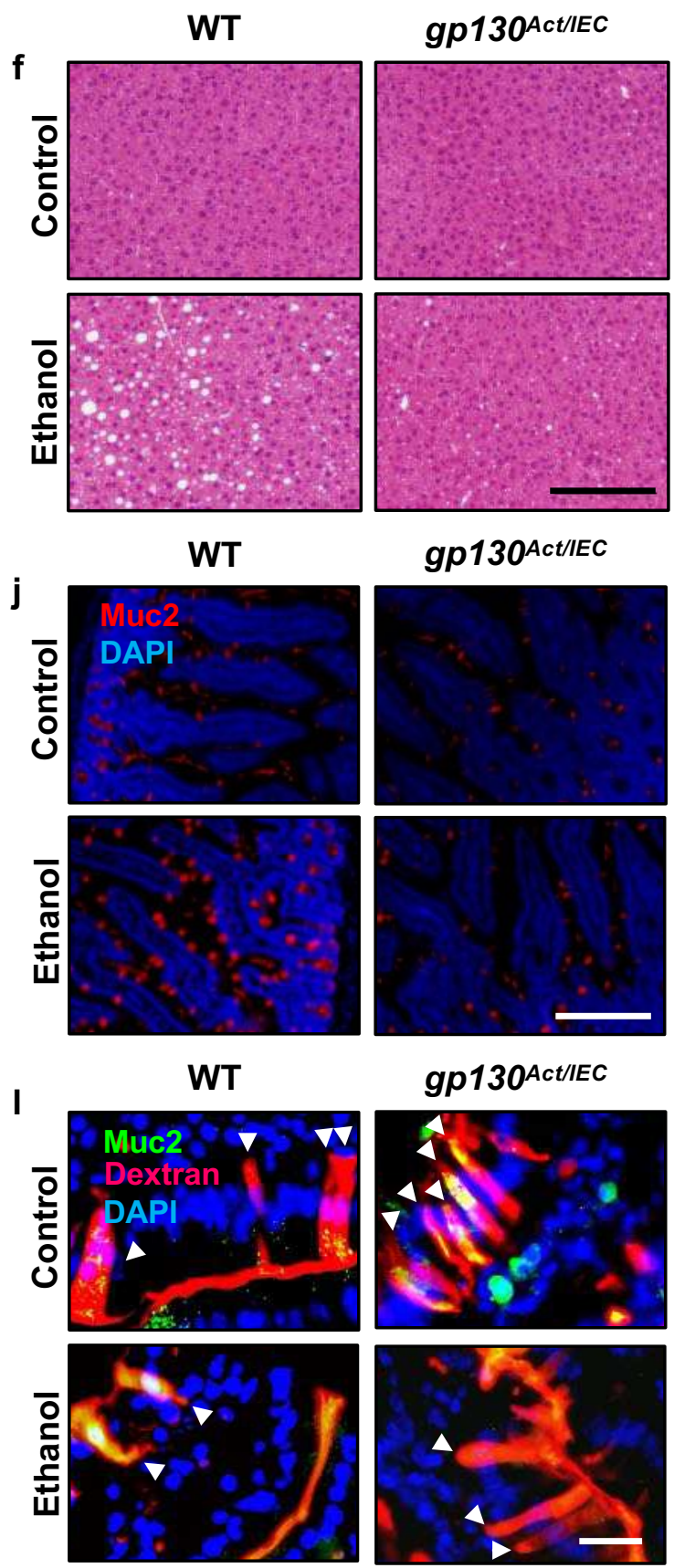

gp130Act/IEC

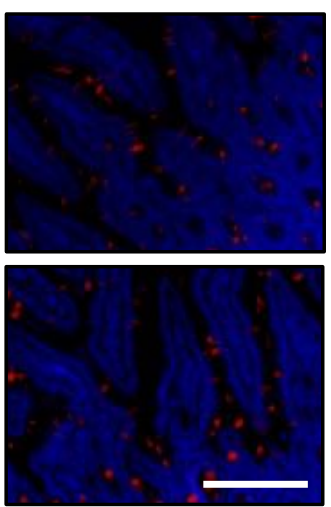

gp130Act/IEC
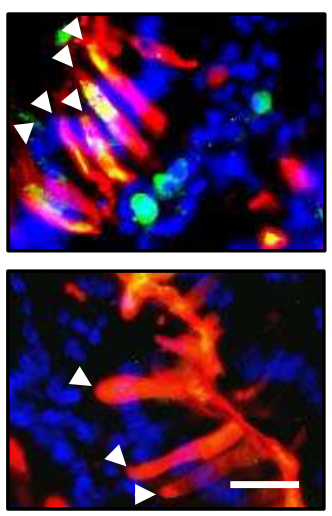

- Isolated goblet cells

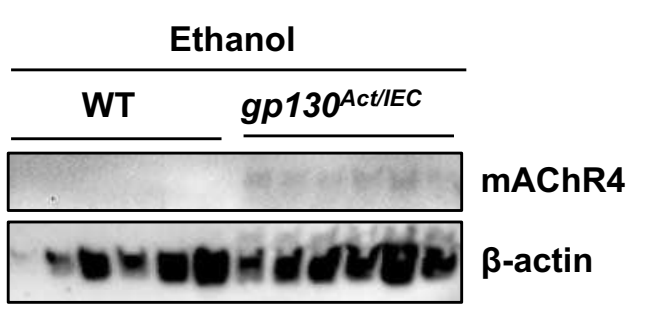

p

Total bacteria

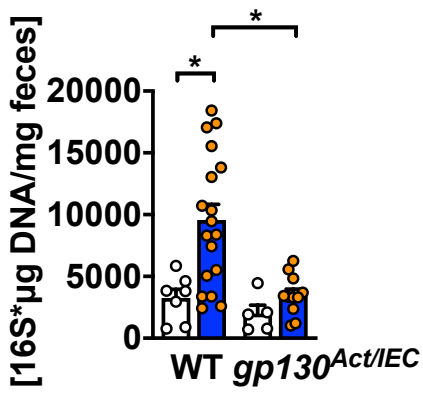

9 PCoA_wunifrac

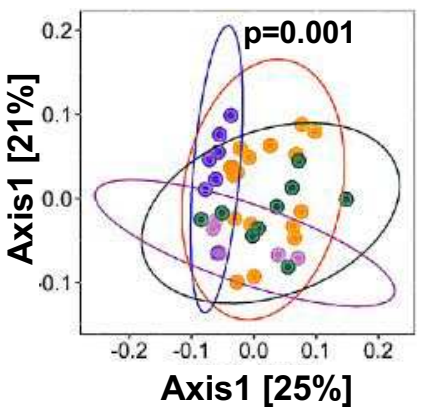

- WT Control

WT Ethanol

gp130 Act/IEC Control gp130Act/IEC Ethanol 
Figure 2. Expression of activated gp130 in IECs prevents ethanol-induced liver disease and promotes GAP formation. (a) Chrm 4 mRNA in small intestinal organoids from WT mice $(\mathrm{n}=2)$ was quantitated by qPCR after stimulation with IL-6 $(0.02 \mu \mathrm{g} / \mathrm{ml})$ in the presence of ethanol (10 $\mathrm{mM}$ ) for 12 h. 6 technical replicates were pooled in each of two independent biological experiments. (b) mAChR4 protein amounts in WT small intestinal organoids after stimulation without ( $\mathrm{n}=3)$ or with IL-6 $(0.5 \mu \mathrm{g} / \mathrm{ml})(\mathrm{n}=3)$ for $24 \mathrm{~h}$. (c) Representative mAChR4 immunoblots. (d-q) WT mice and gp $130^{A c t / I E C}$ littermates were fed control $(\mathrm{n}=4-15)$ or ethanol containing Lieber DeCarli diets ( $\mathrm{n}=4-44)$ for 10 weeks. (d) Plasma levels of ALT. (e) Hepatic triglyceride content. (f) Representative H\&E-stained liver sections. Scale bar $=200 \mu \mathrm{m}$. (g-h) Hepatic Cxcll and Ccl2 mRNAs were quantitated by qPCR. (i) Percentage of Muc2 positive stained area. (j) Representative sections stained with Muc2 antibody (red) showing GCs and DAPI (blue). Scale bar $=200 \mu \mathrm{m}$. (k) Number of GAPs per villus. (l) Representative TMR-dextran (red) showing GAPs, Muc2 antibody (green) and DAPI (blue) stained small intestine sections showing GAPs (Arrowheads). Scale bar $=25 \mu \mathrm{m}$. (m) Small intestinal Chrm4 mRNA was quantified by qPCR. (n) $\mathrm{mAChR} 4$ protein amounts relative to $\beta$-actin in isolated GCs. (o) Immunoblots of mAChR4 and $\beta$-actin in isolated GCs. (p) Total fecal bacteria were quantitated by qPCR. (q) Principal coordinate analysis (PCoA) with weighted UniFrac of $16 \mathrm{~S}$ rRNA sequencing of fecal samples. $P$ values were determined by One-way ANOVA with Tukey's post-hoc test (d, e, i, $\mathrm{k}$ and $\mathrm{p}$ ), by twosided unpaired Student $t$ test or Mann-Whitney U-statistic test (a, b, g, h, m and $\mathrm{n}$ ) and by twosided paired $t$ test. Results are expressed as mean \pm s.e.m. ${ }^{*} P<0.05$. 


\section{Figure 3}

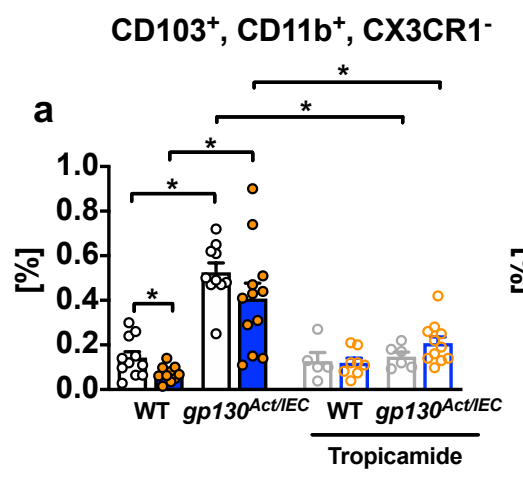

e

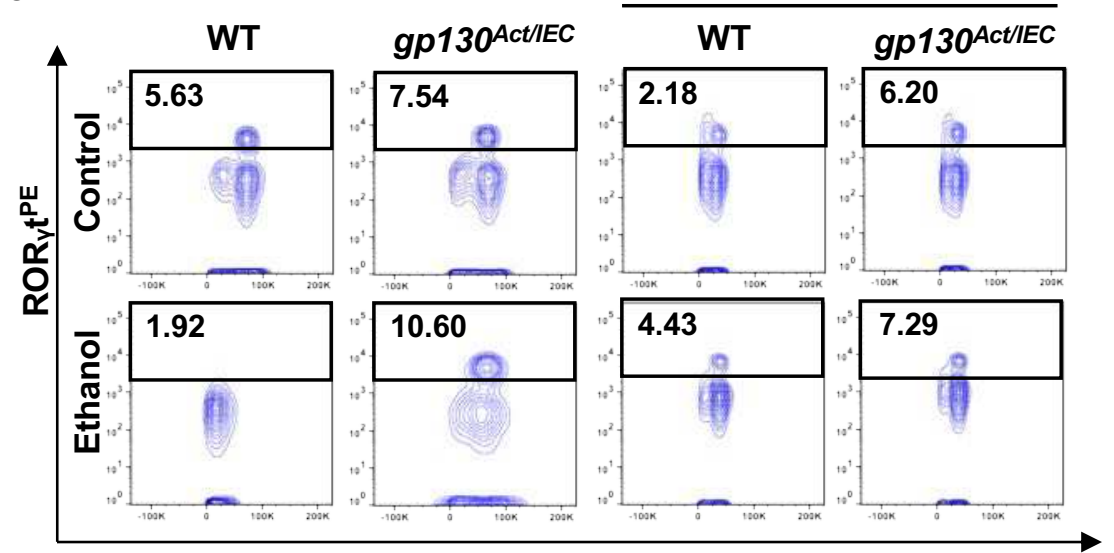

ILC3

j
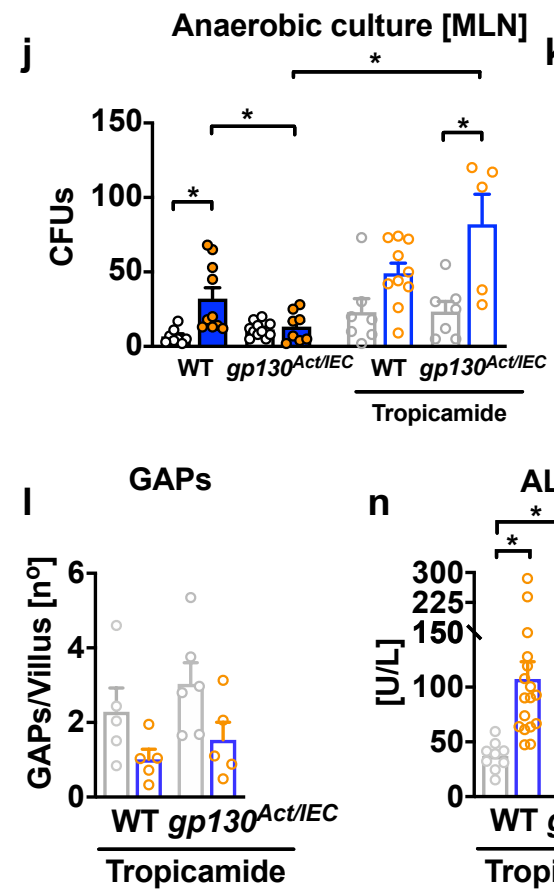
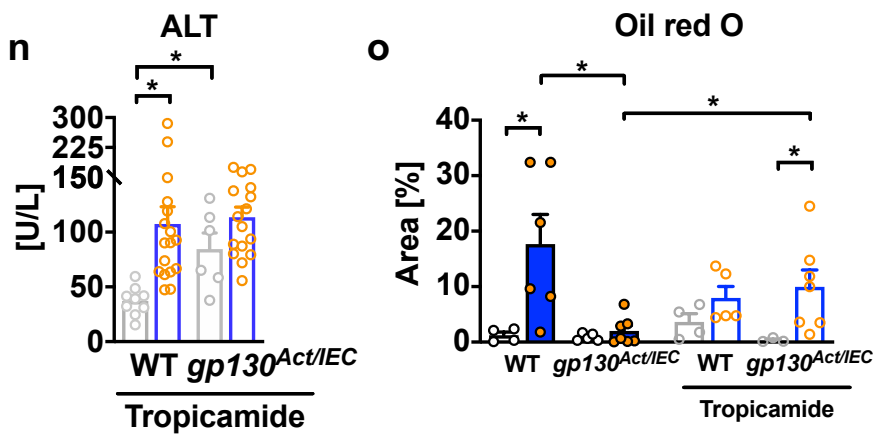

Anaerobic culture [Liver]

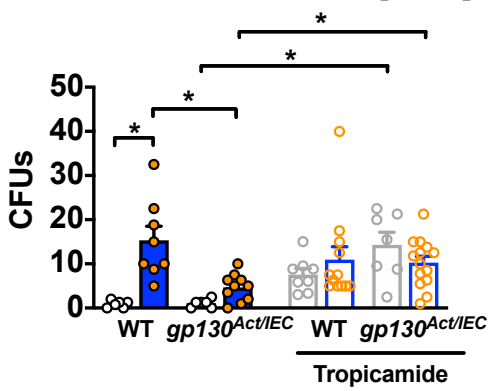

f CD3-, RORyt ${ }^{+}$, IL-22

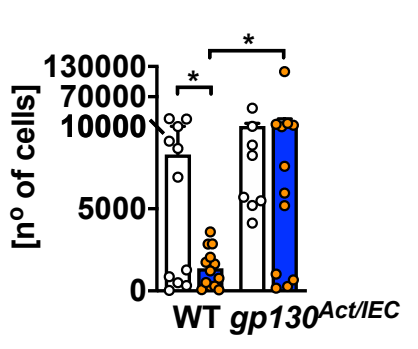

$\mathbf{h}$
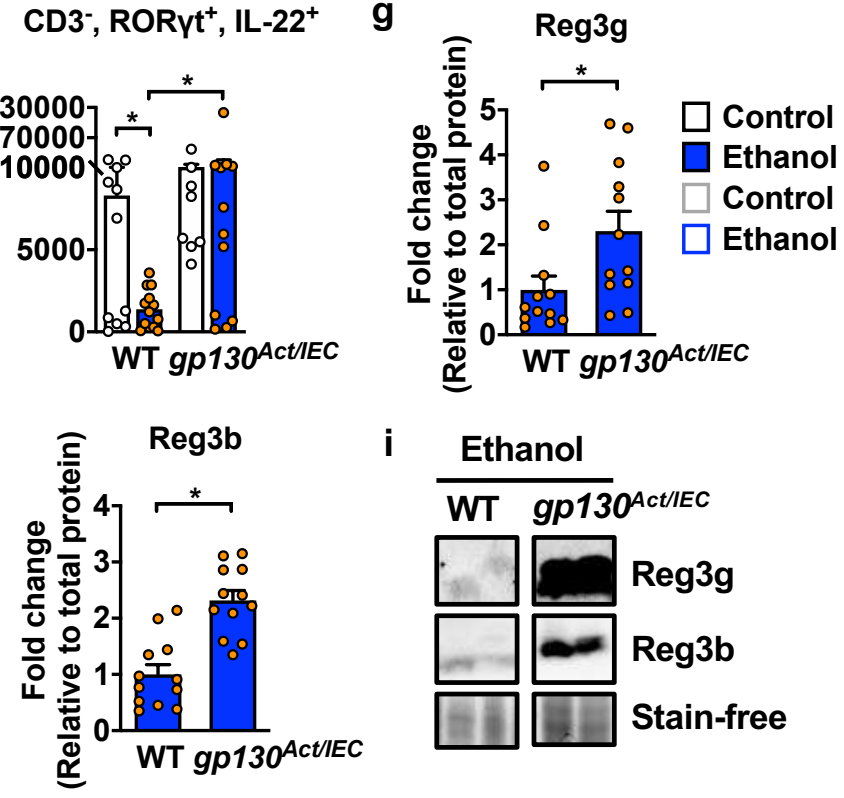

Ethanol

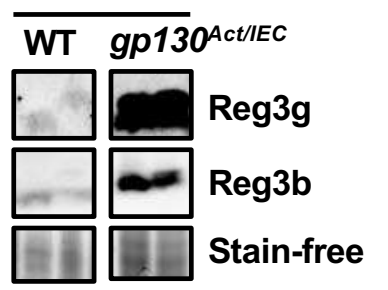

Tropicamide

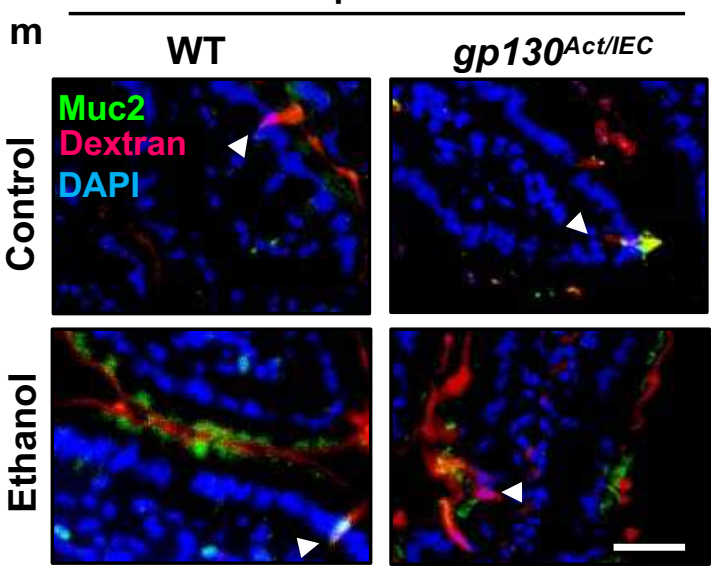

Tropicamide

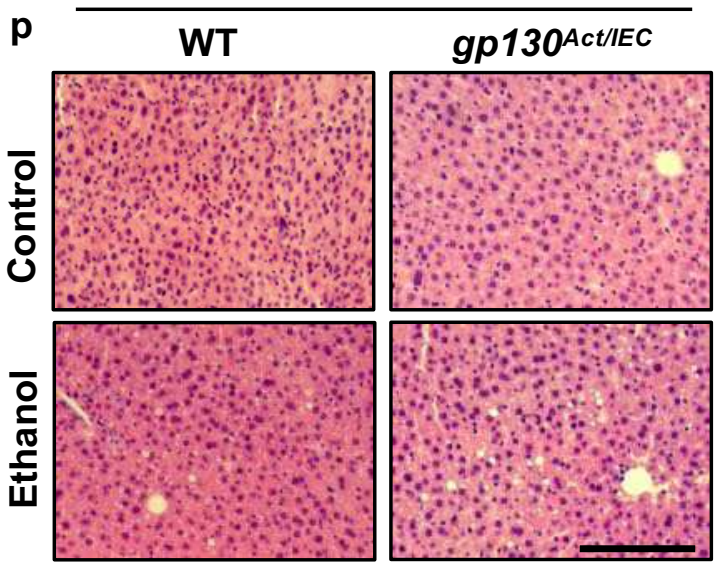


Figure 3. Expression of activated gp130 in IECs stimulates protective intestinal adaptive immune response via mAChR4-mediated GAP formation. (a-t) WT mice and $g p 130^{A c t / I E C}$ littermates were fed control $(n=4-11)$ or ethanol containing Lieber DeCarli diets $(n=6-17)$ for 10 weeks. $(a-d, j-t)$ A group of littermate mice were treated with the mAChR antagonist tropicamide (20 mg/kg) during the last 29 days $(\mathrm{n}=5-17)$. (a-c) Isolated LP immune cells for APC subset identification studies were stimulated with flagellin $(100 \mathrm{ng} / \mathrm{ml})$ for $2.5 \mathrm{~h}$. (a-b) Frequencies of tolerogenic APC subsets in total mononuclear phagocyte population were gated according to CD45, MHCII, CD11c, CD103, CD11b, and CX3CR1 expression. (c) Total number of IL-23 ${ }^{+}$cells in all APCs subsets. (d) Frequencies of ILC3s $\left(\mathrm{CD}^{+} 5^{+}, \mathrm{CD}^{-}, \mathrm{ROR}_{\gamma} \mathrm{t}^{+}\right)$cells after stimulation of isolated LP leukocytes with mouse IL-23 (40 ng/ $\mu$ l) for $4 \mathrm{~h}$. (e) Representative ILC3 and (f) IL-22 expressing ILC3 plots. (g-h) Quantification of Reg3g and Reg3b protein amounts relative to total protein identified by stain-free imaging technology. (i) Representative Reg3g and Reg3b immunoblots. (j, k) Number of colony forming units (CFU) of anaerobically cultured bacteria from sterile collected mesenteric lymph nodes and liver. (1) Number of GAPs per villus. (m) Representative TMR-dextran (red), Muc2 antibody (green) and DAPI (blue) stained small intestinal sections showing GAPs (Arrowheads). Scale bar $=25 \mu \mathrm{m}$. (n) Plasma ALT concentrations. (o) Quantification of the Oil red O (ORO)-stained liver sections. (p) Representative H\&E stained liver sections. Scale bar $=200 \mu \mathrm{m}$. (q) Hepatic Cxcll mRNA quantitated by qPCR. (r, s) Reg3g and Reg3b proteins relative to total protein identified by stain-free imaging technology. (t) Representative Reg3g and Reg3b immunoblots. $P$ values were determined by twoway (a-d, j-k and o) or one-way ANOVA (f, $1, n$ and q) with Tukey's post-hoc test and by twosided unpaired Student $t$ test or Mann-Whitney U-statistic test (g, h, r and s). Results are expressed as mean \pm s.e.m. $* P<0.05$. 
Figure 4

GAPs

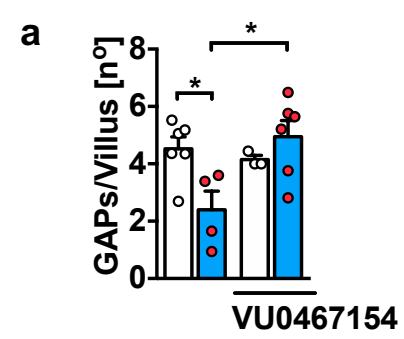

ALT

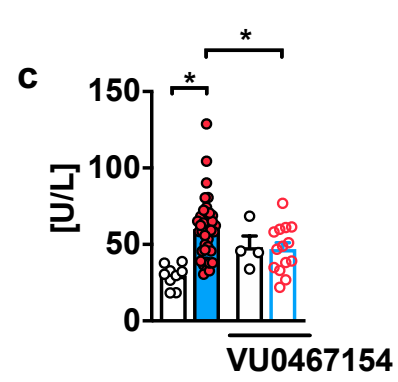

Cxcl1
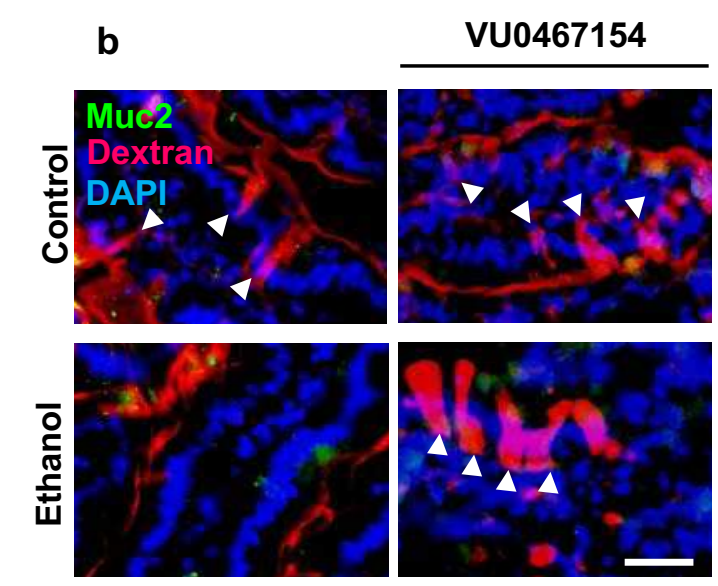

MHC-II+, CD11c ${ }^{+}$

h

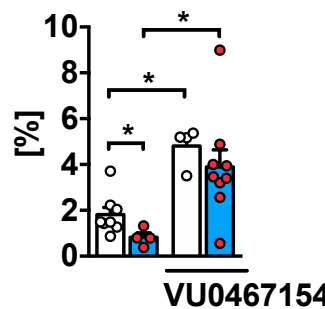

$\mathrm{CD}_{103^{+}}, \mathrm{CD} 11 \mathrm{~b}^{+}$

j
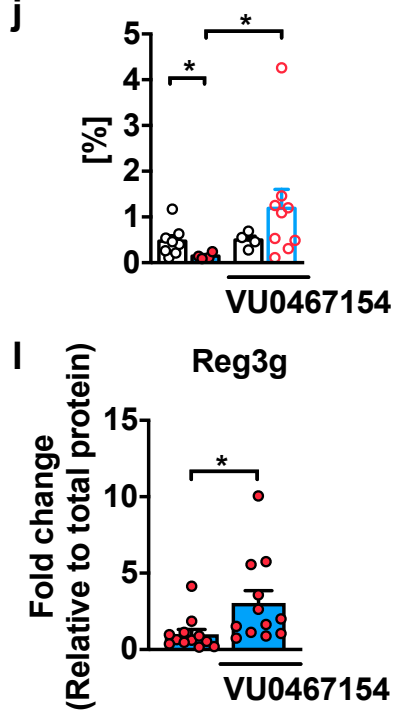

p

Anaerobic culture [MLN]

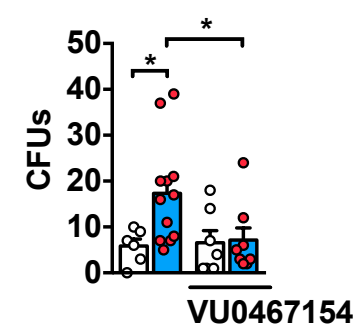

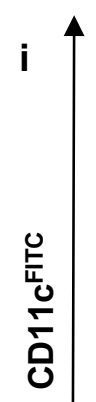

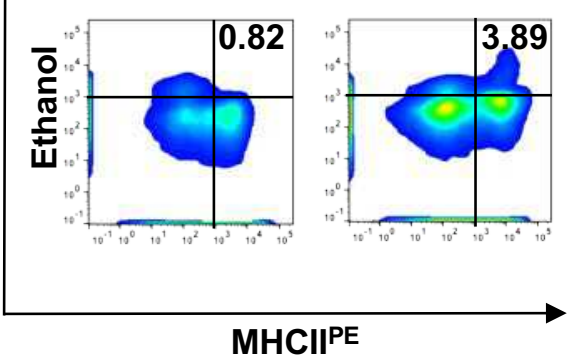

m.
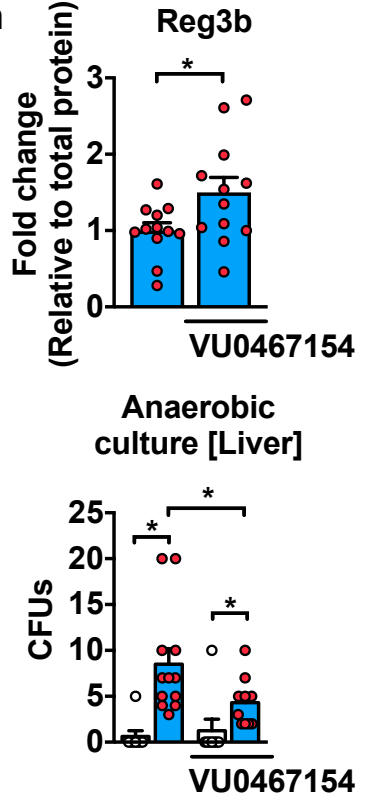

VU0467154

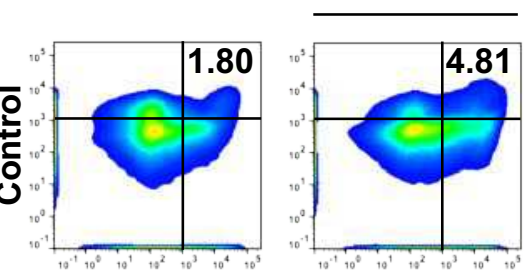

Oil Red O

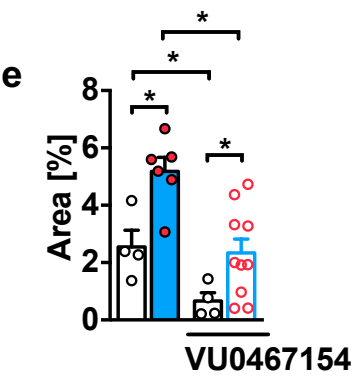

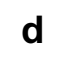
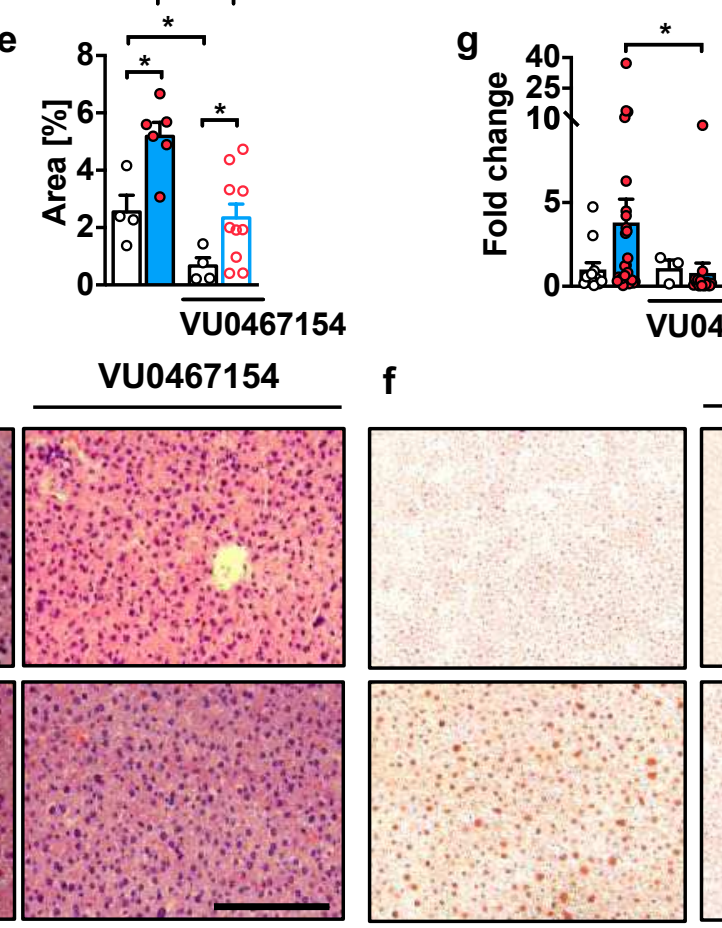

VU0467154
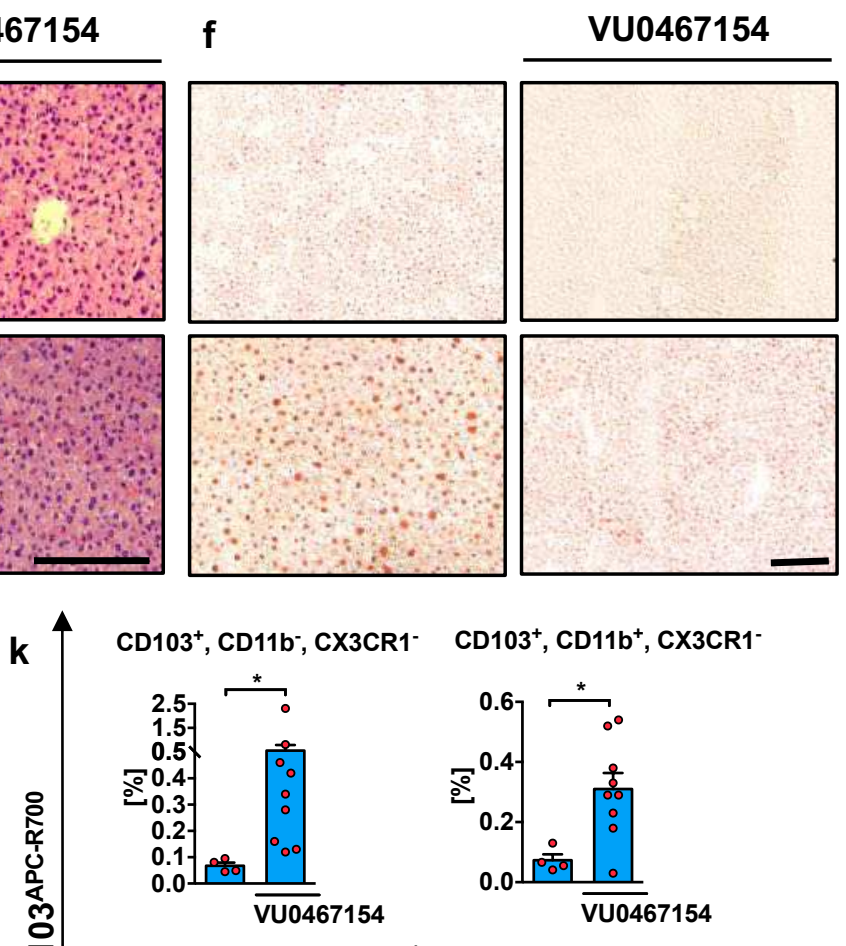

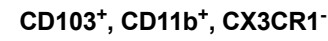

CD103;, CD11b-, CX3CR1 ${ }^{+}$

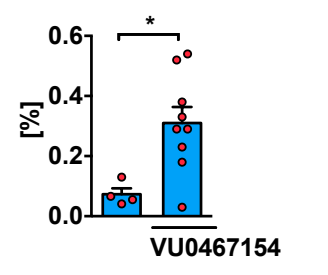

CD103', CD11 ${ }^{+}, \mathrm{CX}^{2} \mathrm{CR} 1^{+}$
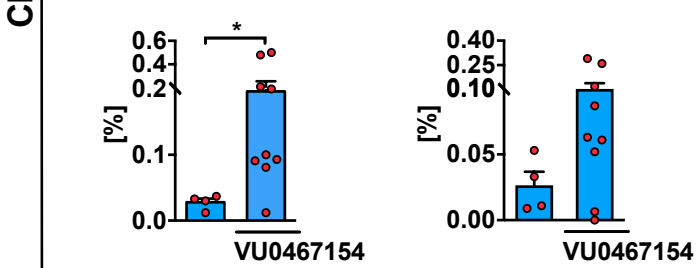

CD11b ${ }^{\text {PerCP-Cy5.5 }}$

n $\overparen{\frac{0}{2}} \quad \mathrm{IL}-10$

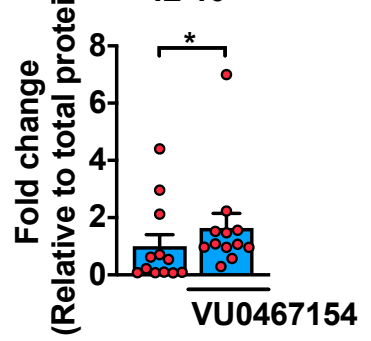

o

Ethanol

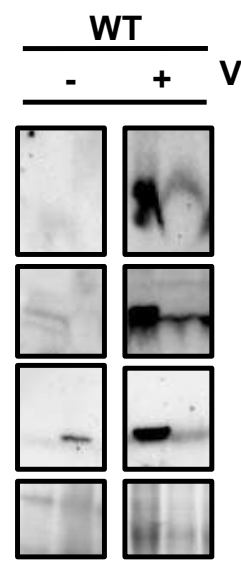

Reg3g

Reg3b

IL-10

Stain-free 
Figure 4. A mAChR4 PAM reduces ethanol-induced liver disease. WT mice were fed control

$414(n=3-9)$ or ethanol containing Lieber DeCarli diets $(n=4-38)$ for 10 weeks in the presence or 415 absence of the specific mAChR4 PAM VU0467154 (5 mg/Kg), dissolved in the diet during the 416 last 29 days. (a) Number of GAPs per villus. (b) Representative TMR-dextran (red), Muc2 (green) 417 and DAPI (blue) stained small intestine sections showing GAPs (Arrowheads). Scale bar $=25 \mu \mathrm{m}$. 418 (c) Plasma ALT concentrations. (d) Representative H\&E stained liver sections. Scale bar $419=200 \mu \mathrm{m}$. (e) Quantification of ORO-stained liver sections. (f) Representative oil ORO-stained 420 liver sections. Scale bar $=100 \mu \mathrm{m}$. (g) Hepatic Cxcll mRNA amounts. (h-k) DCs were stimulated 421 with flagellin $(100 \mathrm{ng} / \mathrm{ml})$ for $2.5 \mathrm{~h}$ before staining. (h) Frequencies of CD45 ${ }^{+}, \mathrm{MHCII}^{+}, \mathrm{CD}_{11 \mathrm{c}^{+}}$ 422 APCs in total mononuclear phagocyte population. (i) Representative APCs plots. (j) Frequencies 423 of $\mathrm{CD}^{2} 5^{+}, \mathrm{MHCII}^{+}, \mathrm{CD} 11 \mathrm{c}^{+}, \mathrm{CD} 103^{+}, \mathrm{CD} 11 \mathrm{~b}^{+}$DCs. (k) Frequencies of tolerogenic APC subsets 424 according to CD45, MHCII, CD11c, CD103, CD11b and CX3CR1 expression. (1-n) 425 Quantification of Reg3g, Reg3b and IL-10 proteins relative to total protein identified by stain-free 426 imaging technology. (o) Representative Reg3g, Reg3b, and IL-10 immunoblots. (p, q) Number of 427 CFUs of anaerobically cultured bacteria from sterile collected MLNs and liver. $P$ values were 428 determined by one-way ANOVA (a, c, e, g, h, j, p, q) with Tukey's post-hoc test and by two-sided 429 unpaired Student $t$ test or Mann-Whitney U-statistic test (k, 1-n). Results are expressed as mean \pm $430 \quad$ s.e.m. $* P<0.05$. 


\section{Methods}

437 Human study. Duodenal biopsies from fifteen patients with AUD and eight biopsies from non438 alcoholic controls were obtained from the alcohol withdrawal unit of Cliniques Universitaires 439 Saint-Luc, Brussels, Belgium where they followed a highly standardized detoxification and 440 rehabilitation program. The demographic data for controls and patients as well as clinical and 441 laboratory characteristics for patients are shown in Extended Data Table 1. AUD patients showed 442 a wide range of liver involvement ranging from minimal liver disease to steatohepatitis or 443 steatofibrosis but no patient had cirrhosis (Extended Data Table 2). Patients were actively drinking 444 until the day of admission. Exclusion criteria included antibiotics use during the two months 445 preceding enrollment, immunosuppressive medication, diabetes, inflammatory bowel disease, 446 known liver disease of any other etiology, and clinically significant cardio-vascular, pulmonary or 447 renal co-morbidities. Written informed consent was obtained from all patients and controls. The 448 study protocol was approved by the Ethics Committee of the Université Catholique de Louvain, in 449 Brussels, Belgium. Duodenal biopsies were obtained during an upper gastro-intestinal endoscopy 450 performed at day 2 of admission. Samples were fixed in $10 \%$ formalin and were paraffin451 embedded. To visualize GCs in humans, we used Periodic Acid/Schiff(PAS) staining. Slides were 452 digitalized using a SCN400 slide scanner (Leica Biosystems, Wetzlar, Germany) at x20 453 magnification and subjected to analysis with the image analysis tool Author version 2017.2 454 (Visiopharm, Hørsolm, Denmark).

456 Mice. C57BL/6 mice were purchased from Charles River and used in Figure 1. WT C57BL/6 mice 457 bred at the UCSD animal facility were used in Figure 4 and Extended Data Figure 5. All mice used 458 in other figures were bred in the same animal facility. gp $130^{A c t / I E C}$ mice on a C57BL/6 background 
459 have been described before ${ }^{7}$. gp $130^{A c t / I E C}$ males and WT littermate females were used for breeding.

460 Littermates were used throughout this study except for ILC3 analysis where 6 non-littermate

461 C57BL/6 WT were included in each group.

462 Female mice (age, 8 weeks) were placed on Lieber DeCarli diet for 10 weeks as previously

463 described ${ }^{8}$. In brief, the Lieber DeCarli diet comprises Micro Stabilized Rod Liq AC IRR

464 (LD101A; TestDiet), Maltodextrin IRR (9598; TestDiet) and 200-proof ethanol (Koptec). The

465 caloric intake from ethanol was 0 on day $1,10 \%$ of total calories on days 2 and 3, 20\% on days 4

466 and 5, 30\% from day 6 until the end of 6 weeks, and 36\% until the end of the treatment. Control

467 mice received an isocaloric amount of iso-maltose instead of ethanol.

468 To study the effect of tropicamide, $g p 130^{A c t / I E C}$ mice and corresponding WT littermate mice fed

469 Lieber DeCarli diet containing ethanol or isocaloric iso-maltose for 10 weeks were treated with

470 the $\mathrm{mAChR} 4$ antagonist tropicamide $(20 \mathrm{mg} / \mathrm{kg})$, which was dissolved in the diet for the last 29

471 days.

472 To enhance GAP formation, WT C57BL/6 mice fed Lieber DeCarli diet containing ethanol or

473 isocaloric iso-maltose for 10 weeks were treated for the last 29 days with the specific mAChR4

474 PAM VU0467154 (5 mg/kg), which was dissolved in the liquid diet.

475 Mice were pair-fed and the amount of liquid diet containing ethanol was similar between mouse

476 strains within each experiment (Extended Data Fig. 1a and Extended Data Fig. 5a). Activation of

477 gp130 in IEC did not affect ethanol absorption (Extended Data Fig. 1b). The use of tropicamide

478 or VU0467154 did not affect ethanol absorption (Extended Data Fig. 1b and Extended Data Fig.

$4795 b)$

480 All animal studies were reviewed and approved by the UCSD Institutional Animal Care and Use 481 Committee. 
483 Bacterial DNA isolation and 16S rRNA sequencing. DNA was isolated from feces of mice. 484 Samples were resuspended in phosphate-buffered saline (PBS) and digested with RNAse A and 485 proteinase $\mathrm{K}$ at $55^{\circ} \mathrm{C}$ for one hour. Each suspension was then transferred to individual Qbiogene 486 lysing matrix B tubes and vortexed using a FastPrep FP120 instrument. The lysate was then 487 extracted twice using Phenol/Chloroform/Isoamyl alcohol, precipitated and washed with ethanol, 488 and the DNA resuspended in TE buffer ${ }^{34-36}$. 16S ribosomal RNA (rRNA) PCR was completed 489 using Illumina adaptor and barcode ligated 16S primers targeting the V4 region of the 16S rRNA 490 gene 9,34,37,38. Amplicons were purified using the Qiaquick PCR purification kit (QIAGEN) 491 following manufacturer's specifications. Purified amplicons were then quantified via TECAN 492 assay (Tecan, Switzerland), normalized, and pooled in preparation for $16 \mathrm{~S}$ rRNA sequencing. The 493 pooled library was quantified and checked for quality using Agilent 2100 Bioanalyzer (Agilent 494 Technologies) and sequenced on Illumina MiSeq (Illumina) using V2 reagent chemistry, 500 495 cycles, 2 x 250bp format using manufacturer's specifications. $16 \mathrm{~S}$ sequence reads were processed 496 and OTUs were determined using our MOTHUR-based 16S rDNA analysis workflow as described 8,34,36. Raw 16S sequence reads can be found in the NCBI SRA associated with Bioproject PRJNA705611 and BioSample IDs: SAMN18094194-SAMN18094231.

Determination of bacterial translocation. Translocation of viable bacteria was assessed by 501 culturing MLNs and liver ${ }^{34}$. Sterile MLNs and liver were homogenized using a bead beater (1.0 502 $\mathrm{mm}$ zirconia/silica beads) under sterile and anaerobic conditions and kept for $1.5 \mathrm{~h}$ at $37^{\circ} \mathrm{C}$ in a 503 bacterial incubator. Different dilutions were plated on CDC Anaerobe 5\% Sheep Blood Agar with 
504 Phenylethyl Alcohol (PEA) plates inside of an anaerobic workstation and cultured anaerobically 505 at $37^{\circ} \mathrm{C}$ for $72 \mathrm{~h}$.

506

507 Staining procedures. Formalin-fixed tissue samples were embedded in paraffin (Paraplast plus, 508 McCornick) and stained with H\&E (Surgipath). To determine lipid accumulation, liver sections 509 were embedded in OCT (Tissue-Tek ${ }^{\mathrm{R}}$ ) compound. $5 \mu \mathrm{m}$ frozen sections were then cut and stained 510 with Oil Red O (Sigma-Aldrich). Representative pictures from each group of mice are shown in 511 each figure. All samples were analyzed by densitometry, using National Institutes of Health (NIH) 512 Image J.

513 For immunofluorescence staining, tissues were fixed in $10 \%$ buffered formalin, embedded in 514 paraffin and sectioned at $5 \mu \mathrm{m}$ thickness and stained with anti-Muc2 (1:200) (San Cruz 515 Biotechnology) primary antibody, or anti-mAChR4 (1:200) (Alomone labs) overnight, or anti516 GFP antibody (1:200) (Abcam) and then, incubated with Alexa fluor 568- or Alexa fluor 488517 conjugated secondary antibodies (Invitrogen). Nuclei were stained in blue with a Vectashield ${ }^{\mathrm{R}}$ 518 (Vector Laboratories) mounting medium containing DAPI and imaged by fluorescent microscopy. 519 Control sections were stained with isotype antibody and showed no staining. All samples were 520 analyzed by densitometry, using NIH Image J.

521 To enumerate GAPs a $2 \mathrm{~cm}$ small intestinal loop was clipped and injected with $200 \mu \mathrm{of} 10 \mathrm{mg} / \mathrm{ml}$ 522 tetramethylrhodamine (TMR)-dextran 10,000 MW, lysine fixable (Thermo Scientific). Next, 523 tissue was fixed in $10 \%$ formalin overnight and subsequently embedded in OCT for frozen 524 sectioning $(5 \mu \mathrm{m})$ for Muc2 immunofluorescence staining as above. Number of GAPs were 525 identified as TMR-dextran-filled columns measuring approximately $20 \mu \mathrm{m}$ (height) $\mathrm{x} 5 \mu \mathrm{m}$ 526 (diameter) traversing the epithelium and containing a nucleus. Small intestinal GAPs were 
527

528

529

530

531

532

533

534 535

536

537

538

539

540

541

542

543

544

545

546

547

35

enumerated as GAPs per villus. To evaluate the intercommunication of bacteria with LP-immune cells via GAPs, $5 \times 10^{9}$ E. faecalis genetically modified with an EGFP vector were gavaged at 3 and $0.5 \mathrm{~h}$ before the end of the study. Trans-epithelial dextran columns did not cause disruption of the epithelial barrier as shown by the exclusion of dextran from the LP.

Bacterial culture. E. faecalis was genetically modified with an EGFP reporter plasmid pBSU101 ${ }^{39}$. EGFP-E. faecalis were grown in brain heart infusion (BHI) broth or on $\mathrm{BHI}$ agar plate at $37^{\circ} \mathrm{C}$ with $125 \mu \mathrm{g} / \mathrm{ml}$ spectinomycin (Sigma). $5 \times 10^{9} \mathrm{CFUs}$ were gavaged as indicated in figure legends.

Real-time quantitative PCR. RNA was extracted from mouse tissues and cDNA was generated

${ }^{9}$. Primer sequences for mouse genes were obtained from the NIH qPrimerDepot. All primers used in this study are listed in Extended Data Table 3. Gene expression was determined with Sybr Green (Bio-Rad Laboratories) using ABI StepOnePlus real-time PCR system. The qPCR value was normalized to $\operatorname{Tbp}$ or $18 \mathrm{~S}$ housekeeping genes. To quantify the total bacterial load in feces, the qPCR value of $16 \mathrm{~S}$ rRNA gene for each sample was multiplied by the total amount of DNA ( $\mu \mathrm{g})$ $\mathrm{mg}^{-1}$ of feces. Published bacterial primer sequences were used for $16 \mathrm{~S}$ rRNA gene ${ }^{40}$ (Extended Data Table 3).

Biochemical analysis. Plasma levels of ALT were determined with Infinity ALT kit (Thermo Scientific). Hepatic triglyceride levels were measured using Triglyceride Liquid Reagents kit (Pointe Scientific). Plasma levels of ethanol were measured using Ethanol Assay kit (BioVision). 
549 Goblet cell isolation. GCs were isolated by selection of biotinylated cytokeratin 18 (CK18)

550 (Abcam) ${ }^{41}$, which is highly expressed in GCs, with streptavidin magnetic beads after isolation of

551 small intestinal cells using a solution containing HBSS without $\mathrm{Ca}^{2+}$ and $\mathrm{Mg}^{2+}, 1 \mathrm{M} \mathrm{HEPES}, 100$

$552 \mathrm{mM}$ sodium pyruvate and 0.5 M EDTA. Magnitude of GC enrichment was confirmed (Extended

553 Data Fig. 1c-1d).

554

555 Small intestinal organoid isolation and culture. Small intestinal crypts were isolated from WT

556 mice, cultured and stained as described ${ }^{42}$ and treated with ethanol (10 mM) and/or IL-6 (0.02, 0.1

557 and $0.5 \mu \mathrm{g} / \mathrm{ml}$ ) (Biolegend) for $12 \mathrm{~h}$ or $24 \mathrm{~h}$.

558

559

Flow cytometry. Isolation: Small intestine was harvested, and the epithelial cell layer was

560 removed. Pieces were digested in media containing $1 \mathrm{mg} / \mathrm{mL}$ collagenase (Millipore Sigma), 0.1

$561 \mathrm{U} / \mathrm{mL}$ dispase (Worthington Biochem) and $0.1 \mathrm{mg} / \mathrm{mL}$ DNase I (Millipore Sigma) at $37^{\circ} \mathrm{C}$ at 150

$562 \mathrm{rpm}$ for $30 \mathrm{~min}$. A $40 \%$ and $80 \%$ Percoll gradient was used to collect the lymphoid fractions at the

563 interphase. Flow cytometry: LP-immune cells were pelleted and blocked with anti-mouse

564 CD16/32 antibody (Thermo Fisher) for 15 min. Cells were divided to perform 3 different panels

565 to analyze APCs, ILC3s and Tregs. Stimulation and staining: To stimulate IL-23 secretion by LP-

566 APCs, cells were re-stimulated in vitro with $100 \mathrm{ng} / \mathrm{ml}$ flagellin (Invivogen) for $2.5 \mathrm{~h}$ in the

567 presence of $0.7 \mu \mathrm{l} / \mathrm{ml} \mathrm{BD} \mathrm{GolgiStop}{ }^{\mathrm{TM}}$ (BD Bioscience) to accumulate cytokines and/or proteins.

568 The murine APC panel consisted of CD45.2 (V500, clone 104, BD), CD11c (FITC, clone N418,

569 ThermoFisher), MHC Class II (I-A/I-E) (PE, clone M5/114.15.2, ThermoFisher), CD1 1b (PerCP-

570 Cy5.5, clone M1/70, BD), CD103 (APC-R700, clone M290, BD), CX3CR1 (BV421, clone 
571 SA011F11, BioLegend), fixable viability stain (FVS) (575V, BD), IL-10 (BV711, clone JES5572 16E3, BD) and IL-23 p19 (AF647, clone N71-1183).

573 In parallel, another set of isolated LP-immune cells was stimulated with $40 \mathrm{ng} / \mu \mathrm{l}$ of recombinant 574 mouse IL-23 (Biolegend) for $4 \mathrm{~h}$ in the presence of $0.7 \mu \mathrm{l} / \mathrm{ml}$ BD GolgiStop ${ }^{\mathrm{TM}}$ (BD Bioscience) to 575 stimulate IL-22 secretion. ILC3 panel consisted of CD45.2 (V500, clone 104, BD), CD3e (FITC, 576 clone 17A2, PharMingen), FVS (575V, BD), IL-22 (APC, clone IL22JOP, ThermoFisher) and 577 ROR $\gamma \mathrm{t}$ (PE, clone B2D, ThermoFisher).

578 Another set of isolated LP-immune cells were stimulated with $10 \mathrm{ng} / \mathrm{ml}$ phorbol 12-myristate 13579 acetate (PMA) (Sigma-Aldrich) plus $500 \mathrm{ng} / \mathrm{ml}$ ionomycin (Sigma-Aldrich) for $4 \mathrm{~h}$ in the presence 580 of $0.7 \mu \mathrm{l} / \mathrm{ml} \mathrm{BD} \mathrm{GolgiStop}{ }^{\mathrm{TM}}$ (BD Bioscience). The third panel consisted of FVS (575V, BD), 581 CD45.2 (V500, clone 104, BD), CD4 (BUV496, clone GK1.5, BD), CD25 (APC-R700, clone 582 PC61, BD), FOXP3 (BV421, clone MF-14, BioLegend).

583 Cells were stained with the corresponding antibodies against the surface receptors of interest for $58430 \mathrm{~min}$ following permeabilization and intracellular staining of cytokines or receptors for $30 \mathrm{~min}$.

585 Cells were recorded using FACS Celesta and LSR Fortessa (BD) flow cytometers at the Flow 586 Cytometry Core Facility at the La Jolla Institute for Immunology (La Jolla, CA). Data was 587 analyzed using FlowJo (version 10.5.3).

588

589 Immunoblot analyses. To measure expression levels of mAChR4, GCs were isolated as described 590 above. Immunoblot analysis was performed as described ${ }^{34}$ using anti-AChR4 1:1000 (\#AMR591 004; Alomone labs) and anti- $\beta$-actin 1:5000 (Sigma-Aldrich) antibody as loading control. Protein 592 from proximal small intestine was extracted and immunoblot analysis was performed using anti593 Reg3g 1:500 (ab198216, Abcam), anti-Reg3b 1:500 (ABIN1870289, Antibodies online), and anti- 
IL-10 1:1000 (sc-365858; Santa Cruz Biotechnology) antibodies. Stain-free imaging technology

595

596

597

598

599

600

601

602

603

604

605

606

607

608

609

610

611

612

613 614 615 616

617

9

\section{1}

\section{3} using polyacrylamide gels (BioRad) containing a proprietary trihalo compound to make proteins fluorescent, was employed to calculate total protein. Immunoblots were visualized with a charged coupling device camera in a luminescent image analyzer (Gel-Doc; Bio-Rad). Immunoblots were analyzed by densitometry, using Image Lab version 6.0.1 software (Bio-Rad).

Data availability. Raw $16 \mathrm{~S}$ sequence reads can be found in the NCBI SRA associated with Bioproject PRJNA705611 and BioSample IDs: SAMN18094194-SAMN18094231.

Statistical analysis. Results are expressed as mean \pm s.e.m. Significance of two groups or multiple groups were evaluated using two-sided unpaired Student's t-test, two-sided unpaired MannWhitney test, or one-way or two-way analysis of variance (ANOVA) with Tukey's post-hoc test, respectively. Statistical analyses were performed using $\mathrm{R}$ statistical software, R v.3.5.1 (R Foundation for Statistical Computing) and GraphPad Prism v9.01. A $P<0.05$ was considered to be statistically significant (adjusted for multiple comparison when performing multiple tests).

(1)

\section{2}




\section{Method references}

34 Yan, A. W. et al. Enteric dysbiosis associated with a mouse model of alcoholic liver disease. Hepatology 53, 96-105, doi:10.1002/hep.24018 (2011).

35 Fouts, D. E., Torralba, M., Nelson, K. E., Brenner, D. A. \& Schnabl, B. Bacterial translocation and changes in the intestinal microbiome in mouse models of liver disease. $J$ Hepatol 56, 1283-1292 (2012).

36 Chen, P. et al. Supplementation of saturated long-chain fatty acids maintains intestinal eubiosis and reduces ethanol-induced liver injury in mice. Gastroenterology 148, 203-214 (2015).

37 Haas, B. J. et al. Chimeric 16S rRNA sequence formation and detection in Sanger and 454pyrosequenced PCR amplicons. Genome Res 21, 494-504, doi:10.1101/gr.112730.110 gr.112730.110 [pii] (2011).

38 Caporaso, J. G. et al. Global patterns of 16S rRNA diversity at a depth of millions of sequences per sample. Proceedings of the National Academy of Sciences of the United States of America 108 Suppl 1, 4516-4522, doi:10.1073/pnas.1000080107 1000080107 [pii] (2011).

39 Aymanns, S., Mauerer, S., van Zandbergen, G., Wolz, C. \& Spellerberg, B. High-level fluorescence labeling of gram-positive pathogens. PLoS One 6, e19822, doi:10.1371/journal.pone.0019822 (2011).

40 Maeda, H. et al. Quantitative real-time PCR using TaqMan and SYBR Green for Actinobacillus actinomycetemcomitans, Porphyromonas gingivalis, Prevotella intermedia, tetQ gene and total bacteria. FEMS Immunol Med Microbiol 39, 81-86, doi:S0928824403002244 [pii] (2003).

41 Knoop, K. A. et al. Microbial antigen encounter during a preweaning interval is critical for tolerance to gut bacteria. Sci Immunol 2, doi:10.1126/sciimmunol.aao1314 (2017).

42 Sato, T. et al. Single Lgr5 stem cells build crypt-villus structures in vitro without a mesenchymal niche. Nature 459, 262-265, doi:10.1038/nature07935 (2009).

\section{Acknowledgments}

This study was supported by the American Association for the Study of Liver Diseases (AASLD) Pinnacle Research Award in Liver Disease (8998GA), by the Southern California Research Center for Alcoholic Liver and Pancreatic Diseases (ALPD) and Cirrhosis (P50 AA011999) funded by the National Institute on Alcohol Abuse and Alcoholism (NIAAA) and its Animal Core facilities, and the Isenberg Endowed Fellowship jointly awarded by the Pilot/Feasibility Program of the San Diego Digestive Diseases Research Center (SDDRC) and the Hellman Family Foundation (P30 DK120515) (to C.L.). This study was supported in part by JSPS KAKENHI (JP 15K21775, JP 20H03758), AMED (PRIME) under Grant Number JP 
18gm6210008/19gm6210008/20gm6210008/21gm6210008 (to K.T.). This study was supported

656 in part by grants from Fond National de Recherche Scientifique Belgium (J.0146.17 and 657 T.0217.18) and Action de Recherche Concertée (ARC), Université Catholique de Louvain, 658 Belgium (to P.S). This study was supported in part by NIH grants U01 027681, R01 CA234128, R37 AI043477 (to M.K.), R01 AA24726, R01 AA020703, U01 AA026939, by Award Number 660 BX004594 from the Biomedical Laboratory Research \& Development Service of the VA Office 661 of Research and Development, and a Biocodex Microbiota Foundation Grant (to B.S.).

662

663

\section{Author Contributions}

664

665

C. L. was responsible for acquisition, analysis and interpretation of data, study concept and design and drafting of the manuscript; R. B., N. C., R. B-C., N. P., L. M., A. A., R. Z. and Y. D provided 666 assistance on data acquisition; B. G. was responsible for $16 \mathrm{~S}$ rRNA sequencing data analysis, K. T. created the gp130 $0^{A c t / I E C}$ mouse model, R. N, D. A. B., and M. K. provided scientific advice and 668 technical support, D. E. F. was responsible for 16S rRNA sequencing, P.S. was responsible for collection of human samples, B.S. and M.K. were responsible for the study concept and design, 670 study supervision and editing the manuscript.

671

672

\section{Declaration of Interests}

673 B.S. has been consulting for Ferring Research Institute, HOST Therabiomics, Intercept

674 Pharmaceuticals, Mabwell Therapeutics, Patara Pharmaceuticals and Takeda. B.S.'s institution 675 UC San Diego has received grant support from Axial Biotherapeutics, BiomX, CymaBay 676 Therapeutics, NGM Biopharmaceuticals, Prodigy Biotech and Synlogic Operating Company. B.S. 677 is founder of Nterica Bio. 
678

679 Materials \& Correspondence

680 Michael Karin, Ph.D., Departments of Pharmacology and Pathology, University of California San

681 Diego, MC0723, 9500 Gilman Drive, La Jolla, CA 92093, USA, Phone 858-534-1361, Fax 858-

682 534-8158, Email karinoffice@ucsd.edu

683 Bernd Schnabl, M.D., Department of Medicine, University of California San Diego, MC0063, 6849500 Gilman Drive, La Jolla, CA 92093, Phone 858-822-5311, Fax 858-822-5370, Email 685 beschnabl@ucsd.edu.

686

687

688

689

690

691

692

693

694

695

696

697

698

699 


\section{Extended Data Figure 1}

a

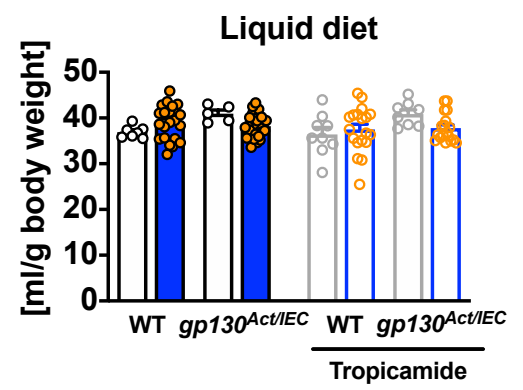

C

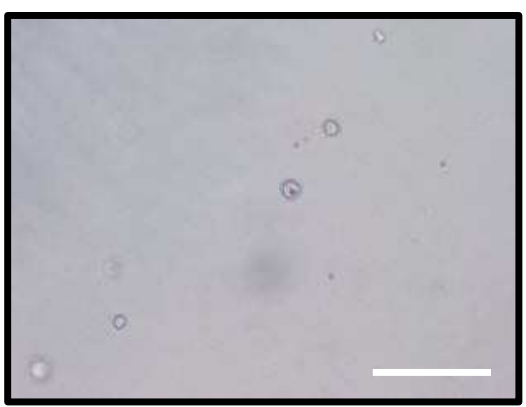

e
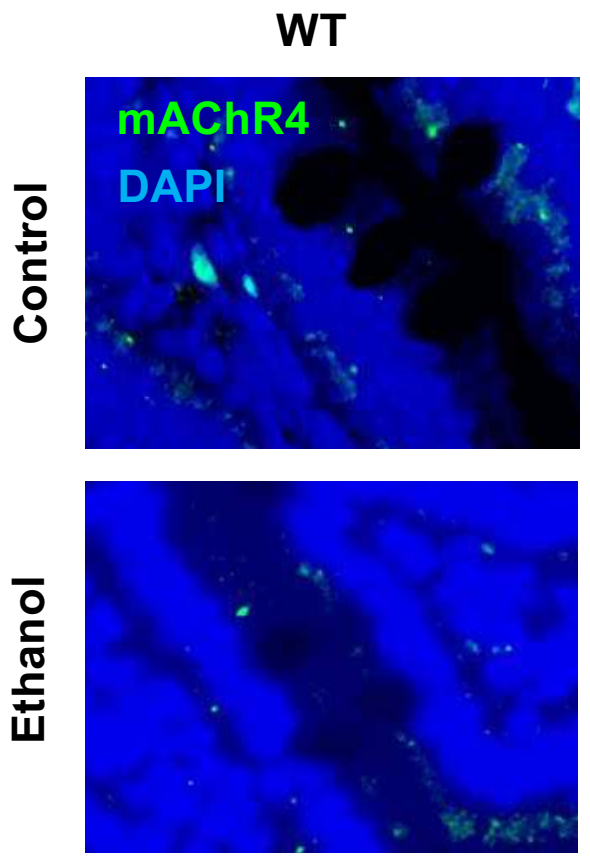

b

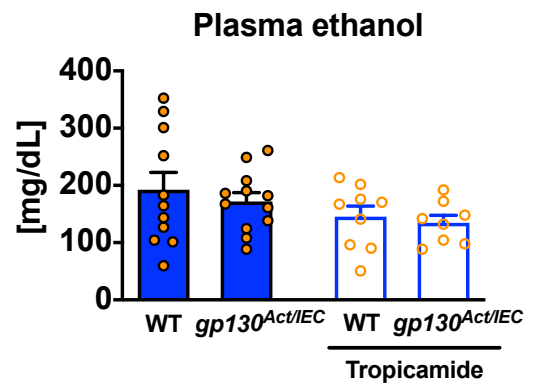

d

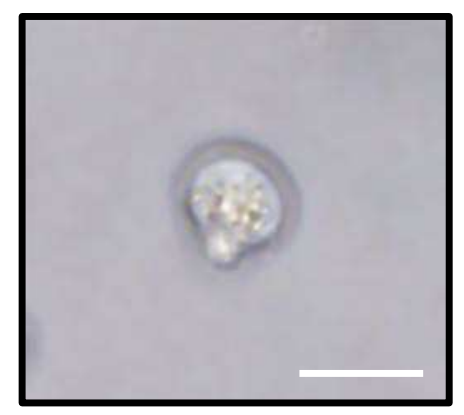

gp130Act/IEC
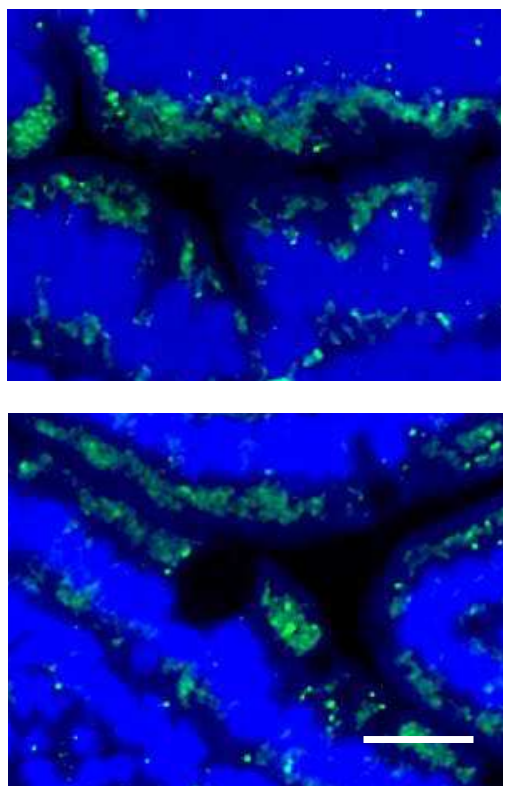

gp130 in IECs after treatments. (a-e) WT mice and their gp130 $0^{A c t / I E C}$ littermates were fed an oral 702 control diet $(n=5-7)$ or ethanol containing Lieber DeCarli diet $(n=11-27)$ for 10 weeks. (a-b) A 703 group of littermate mice were treated with the mAChR4 inhibitor tropicamide $(20 \mathrm{mg} / \mathrm{kg}) \mathrm{during}$ 704 the last 29 days as interventional approach $(n=8-19)$. (a) Food intake of liquid diet. (b) Levels of 705 ethanol in plasma. (c, d) Representative images of isolated goblets cell fraction. (c) Scale bar = $706100 \mu \mathrm{m}$, (d) Scale bar $=20 \mu \mathrm{m}$. (e) Representative sections of mAChR4 (green) and DAPI (blue) 707 immunofluorescence staining showing distribution of the receptors in GCs. Scale bar $=20 \mu \mathrm{m} . P$ 708 values were determined by two-way (a) or one-way ANOVA (b) with Tukey's post-hoc test. 709 Results are expressed as mean \pm s.e.m. $* P<0.05$.

710

711

712

713

714

715

716

717

718

719

720

721 


\section{Extended Data Figure 2}

a

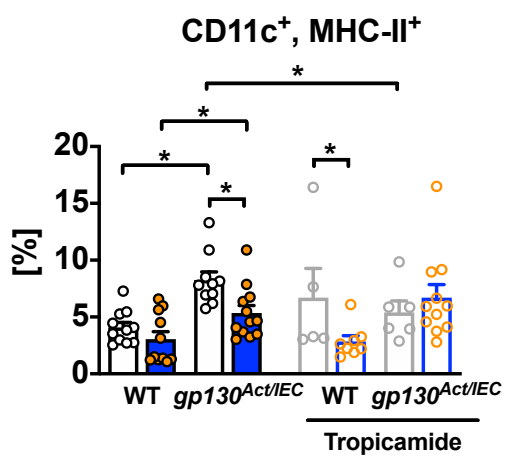

C


b

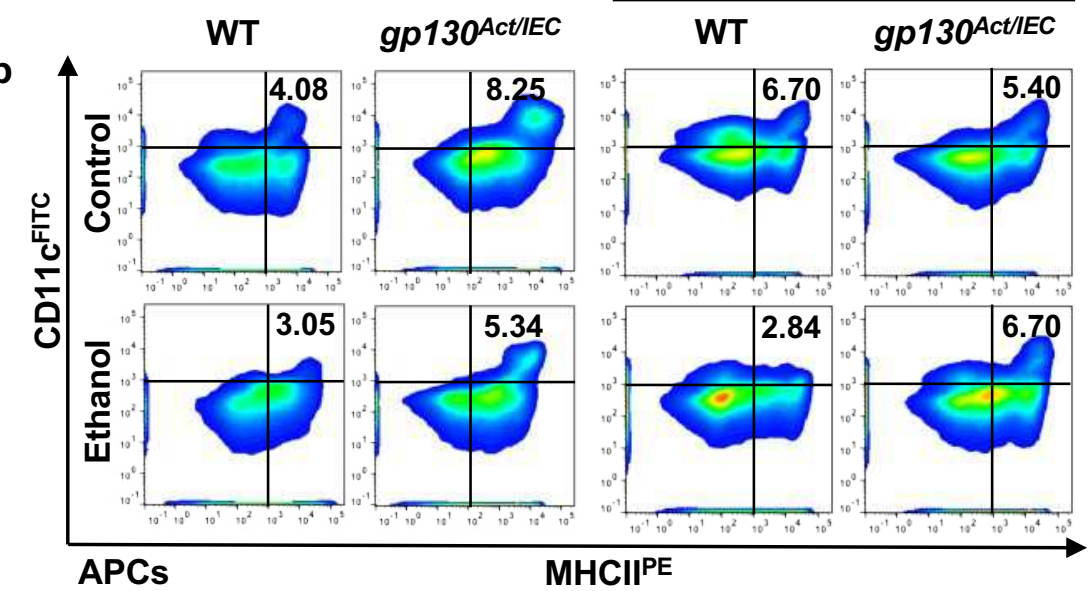

d
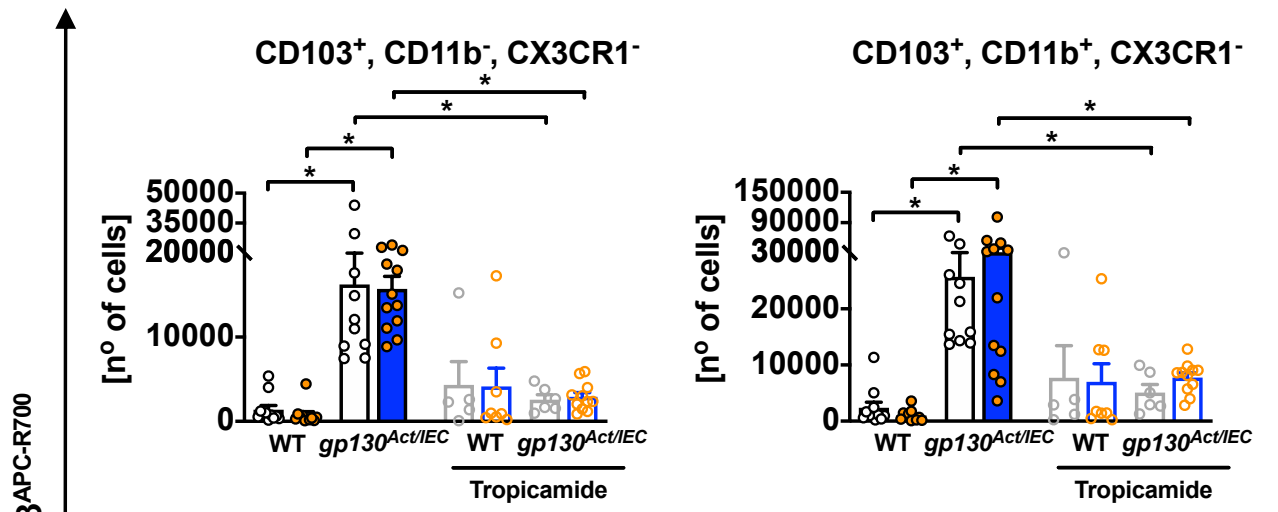

CD103', CD11b", CX3CR1 ${ }^{+}$
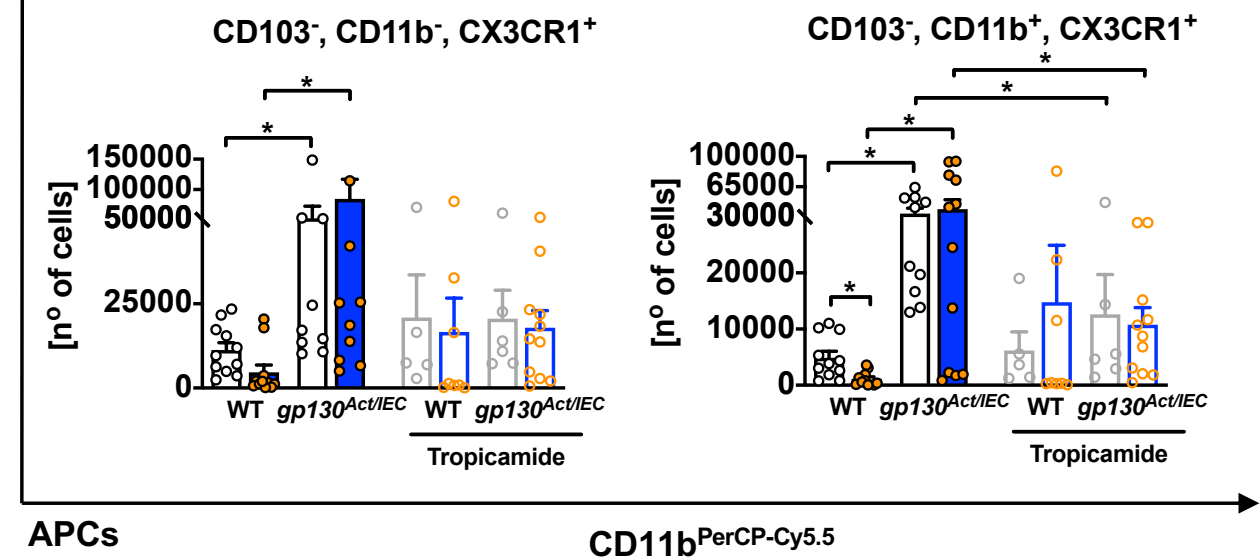

Control

Ethanol

Control

Ethanol

APCs

CD11b ${ }^{\text {PerCP-Cy5.5 }}$ 
722 Extended Data Figure 2. Expression of active gp130 in IECs regulates LP-APCs in small

723 intestine. $(\mathrm{a}-\mathrm{d}) \mathrm{WT}$ mice and their $g p 130^{A c t / I E C}$ littermates were fed an oral control diet $(\mathrm{n}=10-11)$

724 or ethanol containing Lieber DeCarli diet $(n=11-12)$ for 10 weeks. A group of littermate mice

725 were treated with mAChR4 inhibitor tropicamide $(20 \mathrm{mg} / \mathrm{kg})$ during the last 29 days as

726 interventional approach $(n=5-11)$. LP immune cells were isolated and stimulated with flagellin

$727(100 \mathrm{ng} / \mathrm{ml})$ for $2.5 \mathrm{~h}$. (a) Frequencies of $\mathrm{APCs}\left(\mathrm{CD}_{4} 5^{+}, \mathrm{MHCII}^{+}, \mathrm{CD} 11 \mathrm{c}^{+}\right)$at the gate. (b)

728 Representative APCs plots. (c) Total number of APCs $\left(\mathrm{CD} 45^{+}, \mathrm{MHCII}^{+}, \mathrm{CD} 11 \mathrm{c}^{+}\right)$. (d) Total

729 numbers of subsequently gated APCs $\left(\mathrm{CD}^{+} 5^{+}, \mathrm{MHCII}^{+}, \mathrm{CD} 11 \mathrm{c}^{+}\right)$according to $\mathrm{CD} 103, \mathrm{CD} 11 \mathrm{~b}$

730 and CX3CR1 expression markers. (e) Total number of IL-10 expressing cells from all the APCs

731 subsets. $P$ values were determined by two-way ANOVA with Tukey's post-hoc test. Results are

732 expressed as mean \pm s.e.m. $* P<0.05$.

733

734

735

736

737

738

739

740

741

742

743 


\section{Extended Data Figure 3}

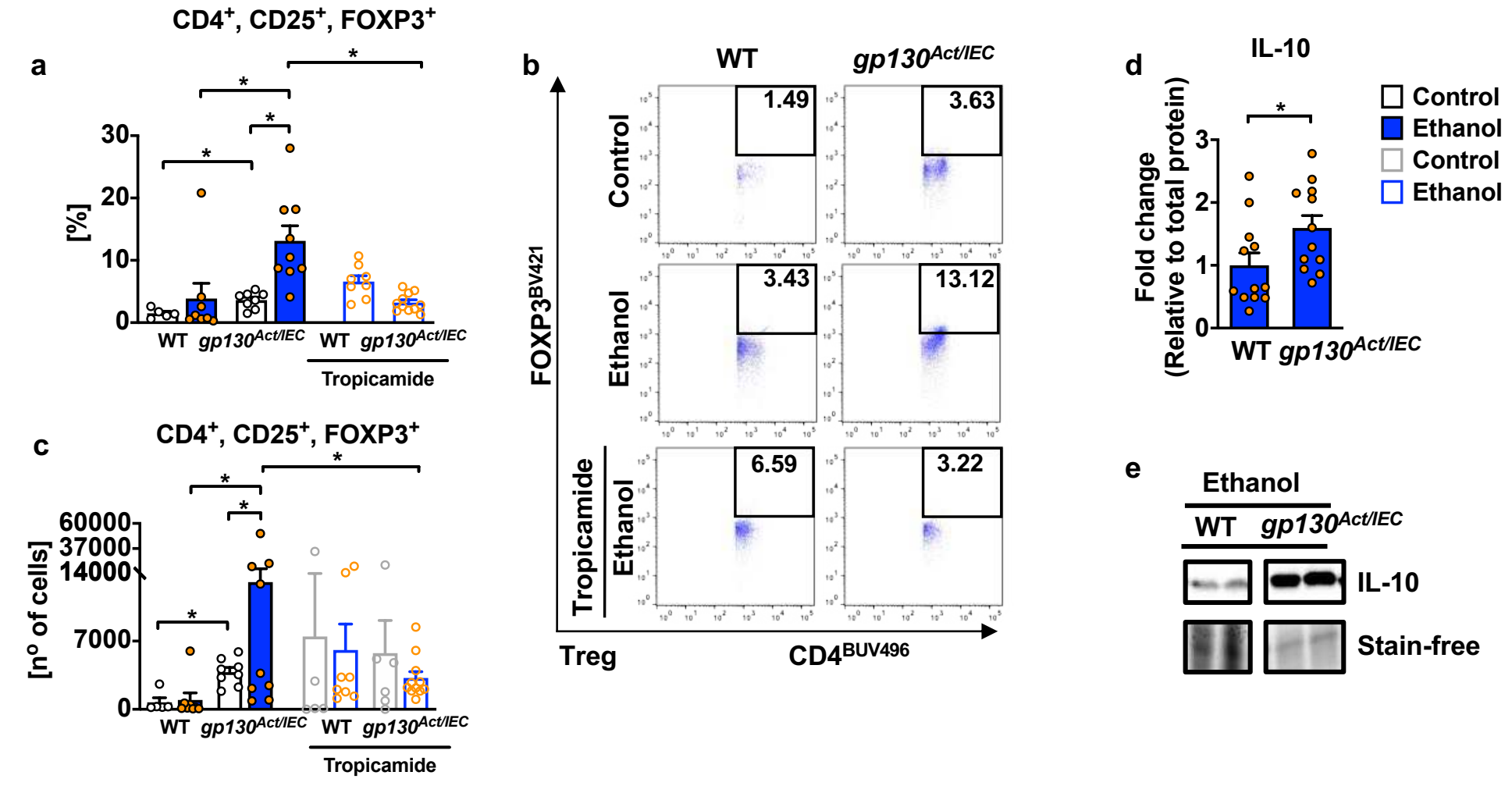


744 Extended Data Figure 3. Expression of active gp130 in IECs regulates LP- Tregs in small

745 intestine. $(\mathrm{a}-\mathrm{d})$ WT mice and their $g p 130^{A c t / I E C}$ littermates were fed an oral control diet $(\mathrm{n}=5-8)$

746 or ethanol containing Lieber DeCarli diet $(n=8-12)$ for 10 weeks. A group of littermate mice were

747 treated with $\mathrm{mAChR} 4$ inhibitor tropicamide $(20 \mathrm{mg} / \mathrm{kg})$ during the last 29 days as interventional

748 approach (n=5-11). (a-c) Cells were stimulated with PMA (10 ng/ml) plus ionomycin $(500 \mathrm{ng} / \mathrm{ml})$

749 for $4 \mathrm{~h}$. (a) Frequencies at the gate of Tregs $\left(\mathrm{CD}^{+}, \mathrm{CD} 25^{+}\right.$and $\left.\mathrm{FOXP} 3^{+}\right)$. (b) Representative Treg

750 plots. (c) Total numbers of Tregs $\left(\mathrm{CD} 4^{+}, \mathrm{CD} 25^{+}\right.$and FOXP3 $\left.{ }^{+}\right)$. (d) Quantification of IL-10 protein

751 levels relative to total protein identified with stain-free imaging technology. (e) Representative IL-

75210 blots. $P$ values were determined by two-way ANOVA with Tukey's post-hoc test (a and c) and

753 by two-sided unpaired Student $t$ test or Mann-Whitney U-statistic test (d). Results are expressed

754 as mean \pm s.e.m. $* P<0.05$.

755

756

757

758

759

760

761

762

763

764

765 


\section{Extended Data Figure 4}
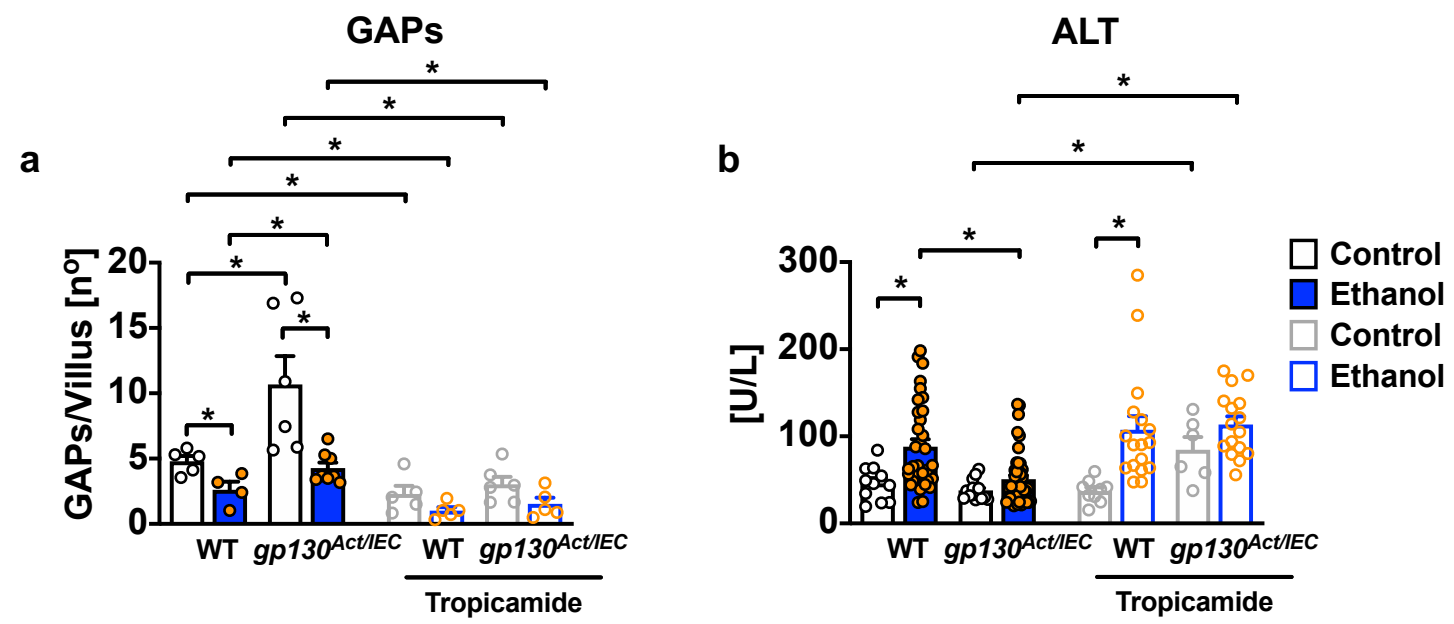

c
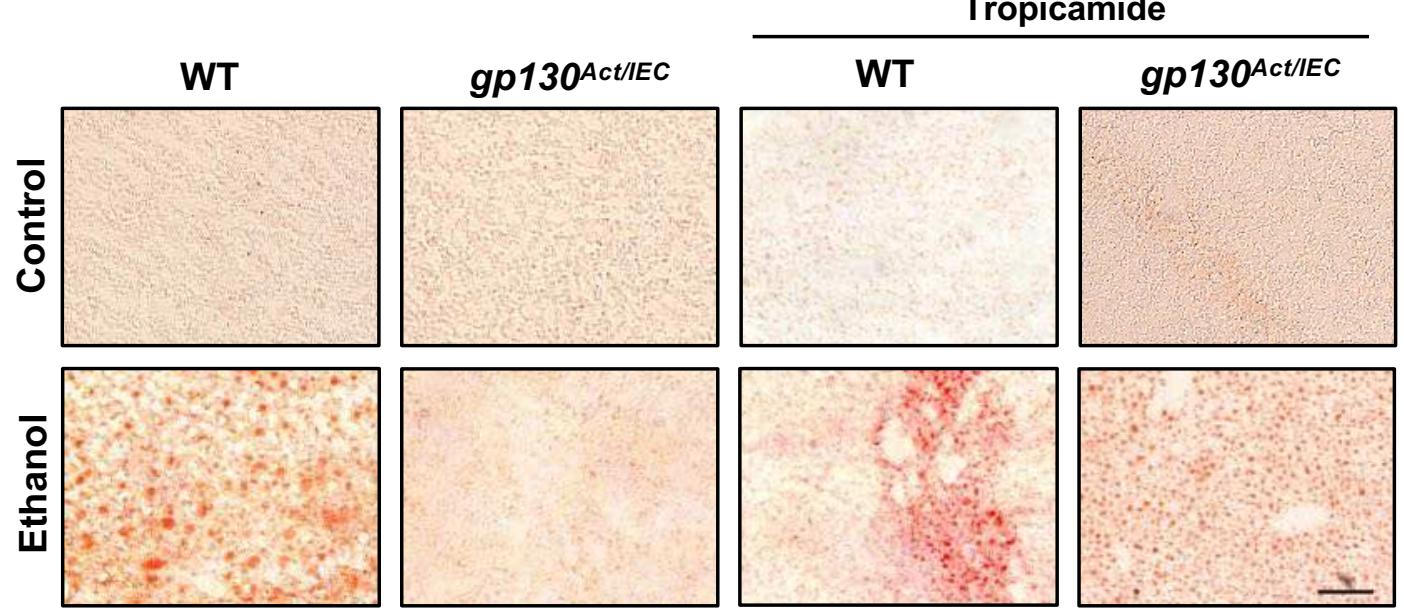

d

Cc/2

e $\quad$ Cxcl5

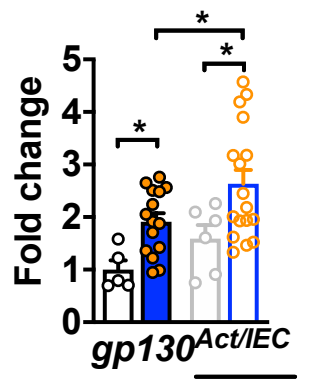

Tropicamide

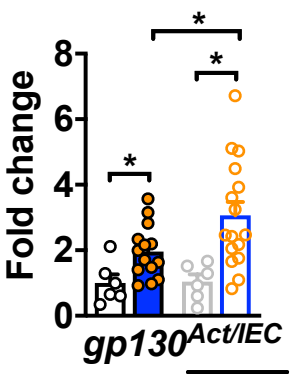

Tropicamide
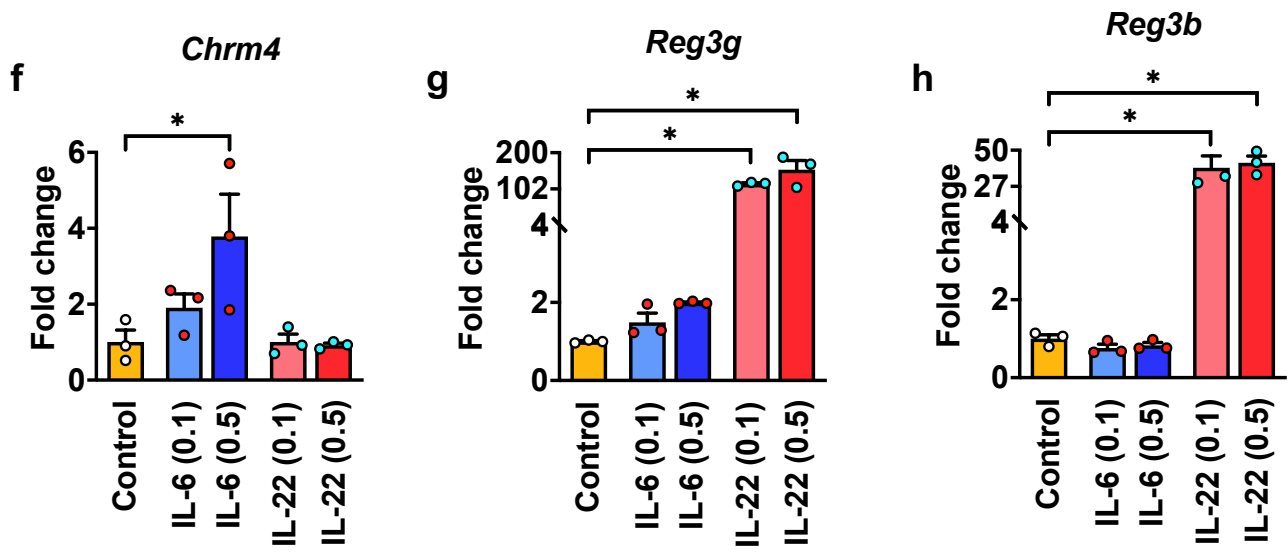
767 the protection against ethanol-induced liver injury in $g p 130^{A c t / I E C}$ mice. (a-e) WT mice and 768 their $g p 130^{A c t / I E C}$ littermates were fed an oral control diet $(\mathrm{n}=5-15)$ or ethanol containing Lieber 769 DeCarli diet $(n=4-44)$ for 10 weeks. (a-e) A group of littermate mice were treated with the $770 \mathrm{mAChR} 4$ inhibitor tropicamide $(20 \mathrm{mg} / \mathrm{kg})$ during the last 29 days as interventional approach $771(\mathrm{n}=6-17) .(\mathrm{a}-\mathrm{b})$ Representation of already described data, now showing all groups in one graph 772 for comparison. (a) Number of GAPs per villus. (b) Plasma levels of ALT. (c) Representative 773 ORO-stained liver sections. Scale bar $=100 \mu \mathrm{m}$. (d-e) Hepatic Ccl2 and Cxcl5 mRNA expression 774 by qPCR. (f-h) Chrm4, Reg3g and $\operatorname{Reg} 3 b$ mRNA in small intestinal organoids from WT mice (n= $7753)$ was quantitated by qPCR after stimulation with IL-6 $(0.1$ and $0.5 \mu \mathrm{g} / \mathrm{ml})$ for $24 \mathrm{~h}$. $P$ values were 776 determined by two-way $(\mathrm{a}, \mathrm{b})$ or one-way ANOVA $(\mathrm{d}-\mathrm{h})$ with Tukey's post-hoc test. Results are 777 expressed as mean \pm s.e.m. $* P<0.05$.

778

779 780 781 782 783 784 785 786 


\section{Extended Data Figure 5}
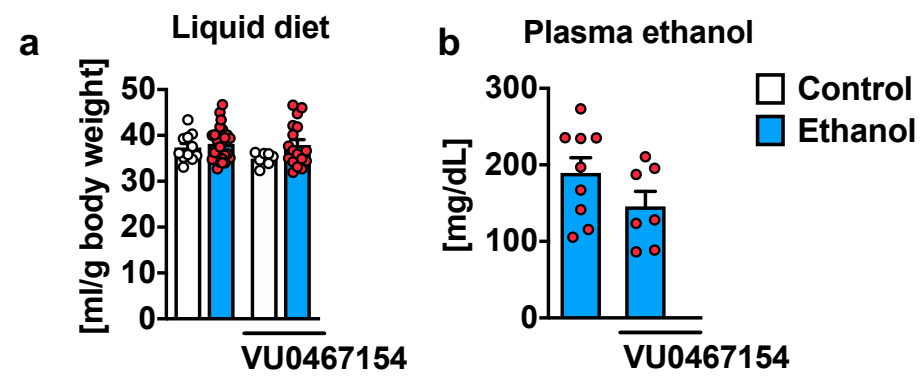

treatment. $(a-b)$ WT mice were fed an oral control diet $(n=7-11)$ or ethanol containing Lieber

790

791

792

793

794 $795 * P<0.05$

796

797

798

799

800

801

802

803

804

805

806

807

808

809

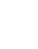

(

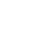

DeCarli diet $(n=7-27)$ for 10 weeks in the presence or absence of a specific mAChR4 positive allosteric modulators, VU0467154 (5 mg/Kg), dissolved in the diet during the last 29 days as interventional approach. (a) Food intake of liquid diet. (b) Levels of ethanol in plasma. $P$ values were determined by one-way ANOVA (a) with Tukey's post-hoc test and by two-sided unpaired Student $t$ test or Mann-Whitney U-statistic test (b). Results are expressed as mean \pm s.e.m. 


\section{Extended Data Figure 6}

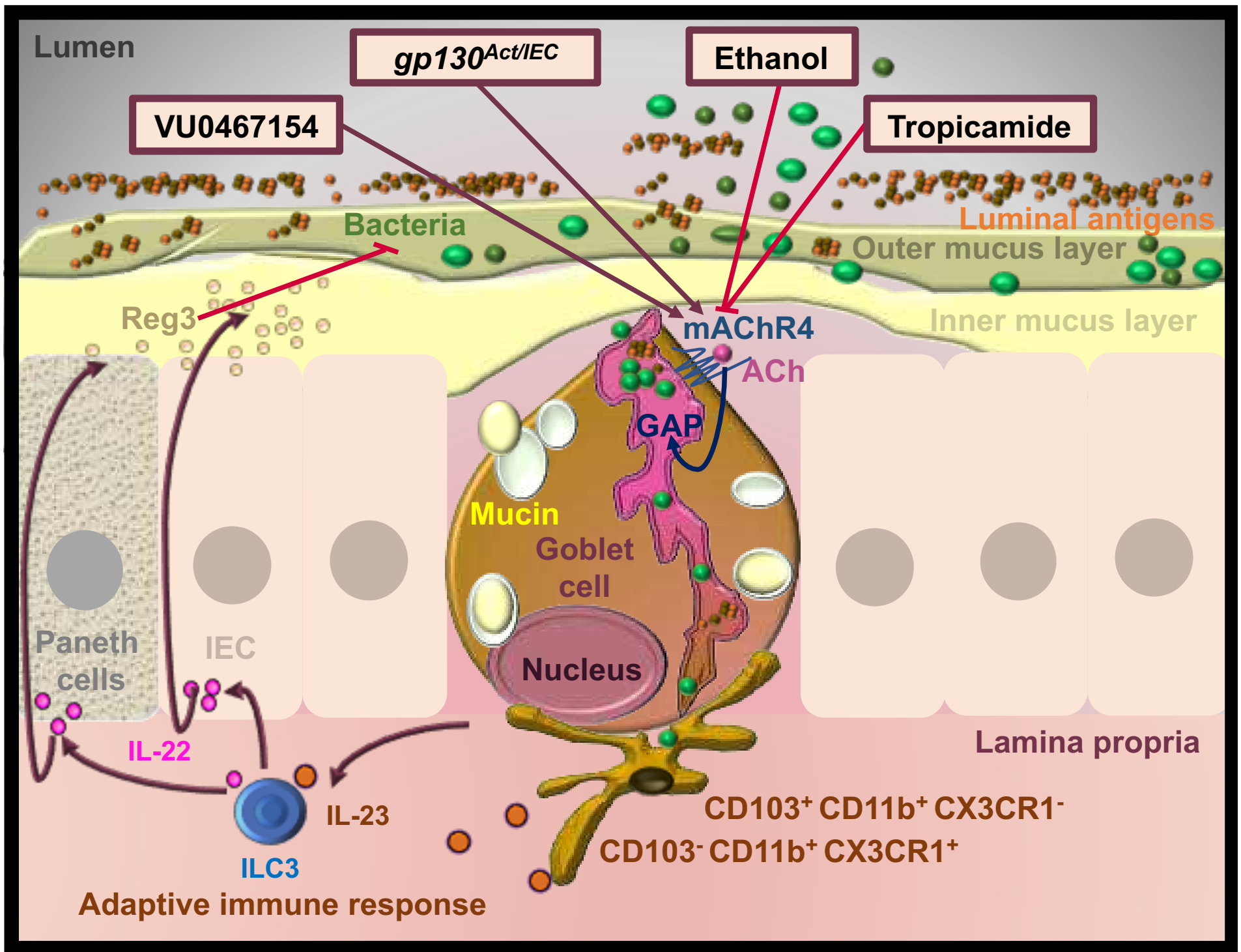


810 Extended Data Figure 6. Graphical abstract. Upon mucin secretion, goblet cells (GCs) form

811 GC associated antigen passages (GAPs) in response to acetylcholine (ACh) acting on muscarinic

812 ACh receptor 4 (mAChR4). GAPs deliver luminal antigens and bacteria to subjacent tolerogenic

$813 \mathrm{CD} 103^{+}, \mathrm{CD} 11 \mathrm{~b}^{+}, \mathrm{CX} 3 \mathrm{CR} 1^{-} \mathrm{DC}$ subset and CD103-, CD11b ${ }^{+}, \mathrm{CX} 3 \mathrm{CR}^{+} \mathrm{APCs}$. Training of these

814 specific LP-APC subsets by GAPs-mediated delivered antigens induces IL-23 secretion which in

815 turn triggers IL-22 production by type 3 innate lymphoid cells (ILC3s) and stimulation of Reg3

816 antimicrobial peptides. This particular adaptive immune response promotes an antibacterial

817 defense, prevents ethanol-induced bacterial translocation to the liver and ameliorates ethanol-

818 induced liver disease. Ethanol-induced suppression of mAChR4 and the mAChR4 antagonist

819 tropicamide inhibit GAP formation. Intestinal IL-6 signal transducer (IL6ST/gp130) signaling and

820 the mAChR4 positive allosteric modulator (PAM), VU0467154, stimulate GAP formation, which

821 offers a promising option to prevent the progression of alcohol-related liver disease through

822 regulation of the intestinal immune response.

823

824

825

826

827

828

829

830

831

832 

patients with alcohol use disorder (AUD).

\begin{tabular}{|c|c|c|}
\hline Variables & $\begin{array}{l}\text { Controls } \\
\qquad(n=8)\end{array}$ & $\begin{array}{l}\text { Alcohol use disorder } \\
\qquad(\mathrm{n}=15)\end{array}$ \\
\hline Gender ( $\%$ male $), \mathrm{n}(\%)$ & $3(37.5)$ & $11(73.3)$ \\
\hline Age (years) & $37.5(24.0-57.0)$ & $44.0(27.0-61.0)$ \\
\hline Weight (kg) & $65(51.0-88.0)$ & $72.0(49.0-107.5)$ \\
\hline BMI (kg/m2) & $22.3(19.8-28.1)$ & $22.4(18.0-33.9)$ \\
\hline Bilirubin (mg/dL) & & $0.4(0.2-3.0)$ \\
\hline AST (IU/L) & & $36.0(14.0-273.0)$ \\
\hline $\operatorname{ALT}(\mathrm{IU} / \mathrm{L})$ & & $37.0(14.0-133.0)$ \\
\hline Albumin $(\mathrm{g} / \mathrm{L})$ & & $48.0(32.0-57.5)$ \\
\hline INR & & $0.9(0.9-1.2)$ \\
\hline GGT (IU/L) & & $120.0(11.0-3460.0)$ \\
\hline ALP (IU/L) & & $83.0(41.0-355.0)$ \\
\hline CAP & & $272.0(208.0-382.0)$ \\
\hline FibroScan (Kpa) & & $6.1(3.1-72.8)$ \\
\hline
\end{tabular}

835

Median values are represented with range in parentheses for continuous variables or percentage in

837 parentheses for categorical variables. Percentages are calculated based on the number of patients 838 in each group.

839

840 ALP, alkaline phosphatase; ALT, alanine aminotransferase; AST, aspartate aminotransferase;

841 AUD, alcohol use disorder; BMI, body mass index; CAP, Controlled attenuation parameter; GGT, 842 gamma-glutamyl transferase; INR, international normalized ratio. 
846

847

848

849

850

851

852

853 Percentages are represented in parentheses.

\section{Alcohol use disorder}

\section{Liver disease severity}

No liver disease, n (\%) $3(20)$

Steatosis, n (\%) $5(33.3)$

Steatohepatitis, n (\%) $2(13.3)$

Steatofibrosis, n (\%) $3(20)$

\begin{tabular}{l|l} 
Unclassified, $\mathrm{n}(\%)$ & $2(13.3)$
\end{tabular}

854

855

856

857

858

859

860

861

862

863

864

865

866

867 


\begin{tabular}{|c|c|c|}
\hline Gene & primer & sequence \\
\hline \multirow[t]{2}{*}{ Mouse $18 S$} & $\mathrm{~F}$ & 5'-AGTCCCTGCCCTTTGTACACA-3' \\
\hline & $\mathrm{R}$ & 5'-CGATCCCAGGGCCTCACTA-3' \\
\hline \multirow[t]{2}{*}{ Mouse Chrm4 } & $\mathrm{F}$ & 5'-ATGGCGAACTTCACACCTGTC-3' \\
\hline & $\mathrm{R}$ & 5'-CTGTCGCAATGAACACCATCT-3' \\
\hline \multirow[t]{2}{*}{ Mouse Cxcll } & $\mathrm{F}$ & 5'-TGCACCCAAACCGAAGTC-3' \\
\hline & $\mathrm{R}$ & 5'-GTCAGAAGCCAGCGTTCACC-3' \\
\hline \multirow[t]{2}{*}{ Mouse $\mathrm{Ccl}$} & $\mathrm{F}$ & 5'-ATTGGGATCATCTTGCTGGT-3' \\
\hline & $\mathrm{R}$ & 5'-CCTGCTGTTCACAGTTGCC-3' \\
\hline \multirow[t]{2}{*}{ Mouse $\mathrm{Cxcl5}$} & $\mathrm{F}$ & 5'-TGATCCCTGCAGGTCCACA-3' \\
\hline & $\mathrm{R}$ & 5'-CTGCGAGTGCATTCCGCTTA-3' \\
\hline \multirow[t]{2}{*}{ Mouse $\underline{T b p}$} & $\mathrm{~F}$ & 5'-GTGAAGGGTACAAGGGGGTG-3' \\
\hline & $\mathrm{R}$ & 5'-ACATCTCAGCAACCCACACA-3' \\
\hline \multirow[t]{2}{*}{ Bacteria 16S } & $\mathrm{F}$ & 5'-GTG STG CAY GGY TGT CGT CA-3' \\
\hline & $\mathrm{R}$ & 5'-ACG TCR TCC MCA CCT TCC TC-3' \\
\hline
\end{tabular}

870

871

872

873

874

875

876 


\section{Figures}

a

Muc2

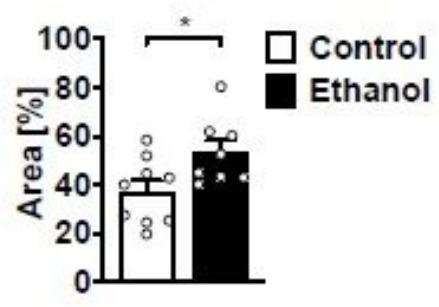

C

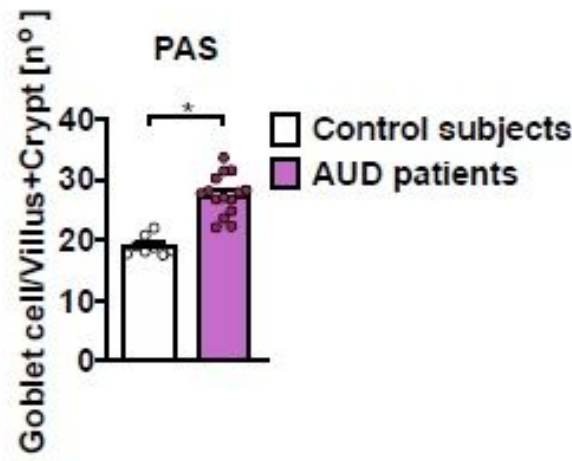

b

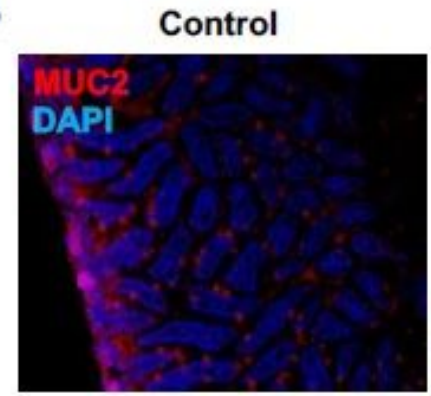

d

Control subjects

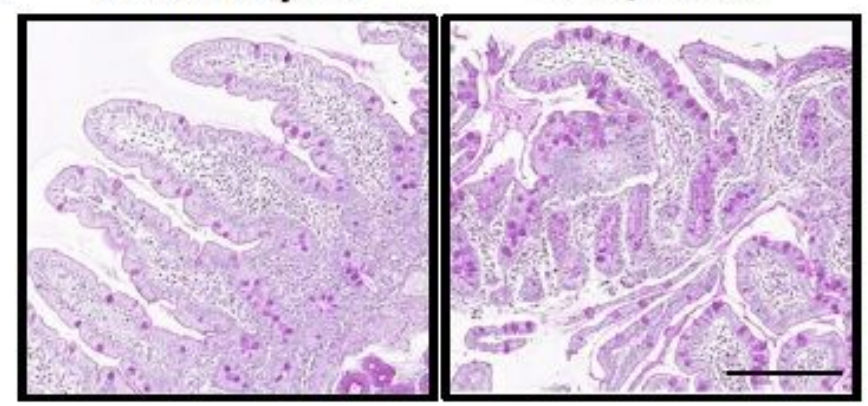

e
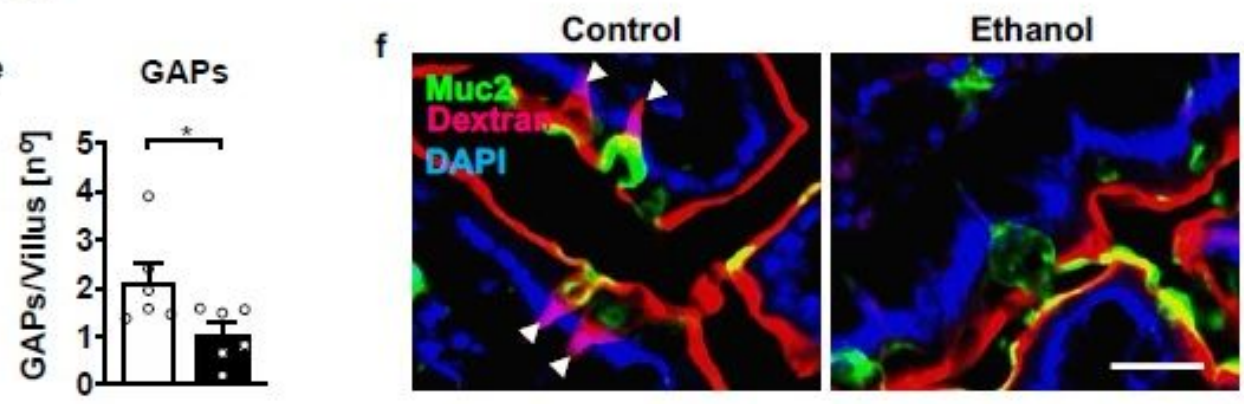

h
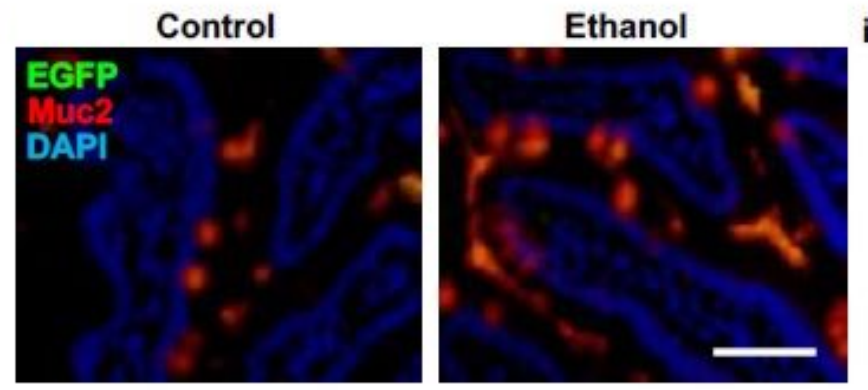

g

Chrm4

Ethanol

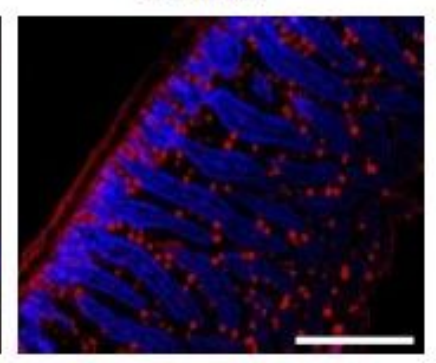

AUD patients

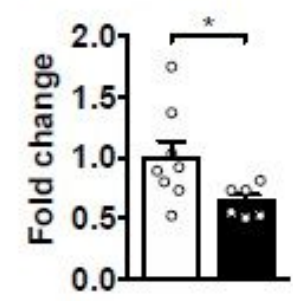

\section{Figure 1}

Chronic ethanol alters proximal intestinal GC in mice 344 and humans. ( $a-b$ and e-i) C57BL/6 WT mice were fed control $(n=6-9)$ or ethanol containing Lieber DeCarli diets $(n=6-8)$ for 10 weeks. (a) Percentage of Muc2 positive stained area. (b) Representative sections of Muc2 (red) showing GCs and DAPI (blue) 
immunofluorescence staining. Scale bar $=250 \mu \mathrm{m}$. (c, d) Periodic Acid/Schiff (PAS) staining of paraffinembedded small intestinal sections of duodenal biopsies from non-alcoholic controls $(n=8)$ and patients with AUD ( $n=15)$. (c) Positive cells were enumerated in each villus and crypt. (d) Representative PASstained sections showing increased number of GCs in the duodenum of AUD patients as compared with controls. Scale bar $=100 \mu \mathrm{m}$. (e) To study GC GAP formation, a $2 \mathrm{~cm}$ loop in the small intestine was injected with tetramethylrhodamine (TMR) dextran and GAPs were counted as dextran-filled columns traversing the nucleated epithelium and positive for Muc2 staining. Number of GAPs per villus was quantified. (f) Representative sections stained with TMR-dextran (red) showing GAPs, Muc2 antibody (green) showing GCs and DAPI (blue) marking nuclei. Scale bar $=25 \mu \mathrm{m}$. (g) Chrm4 mRNA was quantified by qPCR. (h) E. faecalis genetically modified with an EGFP vector $(5 \times 109$ CFUs) were gavaged $9 \mathrm{~h}$ and $1 \mathrm{~h}$ before euthanasia $(\mathrm{n}=6)$. Representative sections stained with Muc2 antibody (red), EGFP (green) and DAPI (blue). Scale bar = $50 \mu \mathrm{m}$. (i) EGFP-E. faecalis ( $5 \times 109 \mathrm{CFUs}$ ) were gavaged at 3 and $0.5 \mathrm{~h}$ before euthanasia $(n=6)$. Representative section of EGFP (green), TMR-dextran (red) and DAPI (blue) stained intestinal sections. Left panel: scale bar $=100 \mu \mathrm{m}$, and right panel (amplification of the white discontinuous oval): scale bar $=12 \mu \mathrm{m}$. ( $\mathrm{f}$ and $\mathrm{i}$ ) Arrowheads indicate GAPs. P value was determined by two-sided unpaired Student $t$ test or Mann-Whitney U-statistic test. Results are expressed as mean \pm s.e.m. ${ }^{*} P<0.05$. 

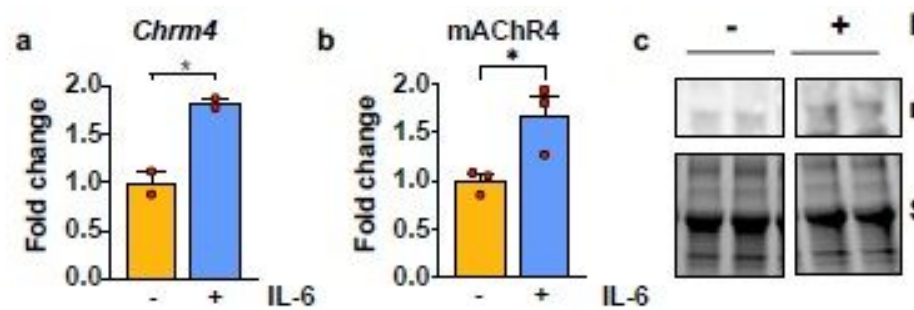

IL-6
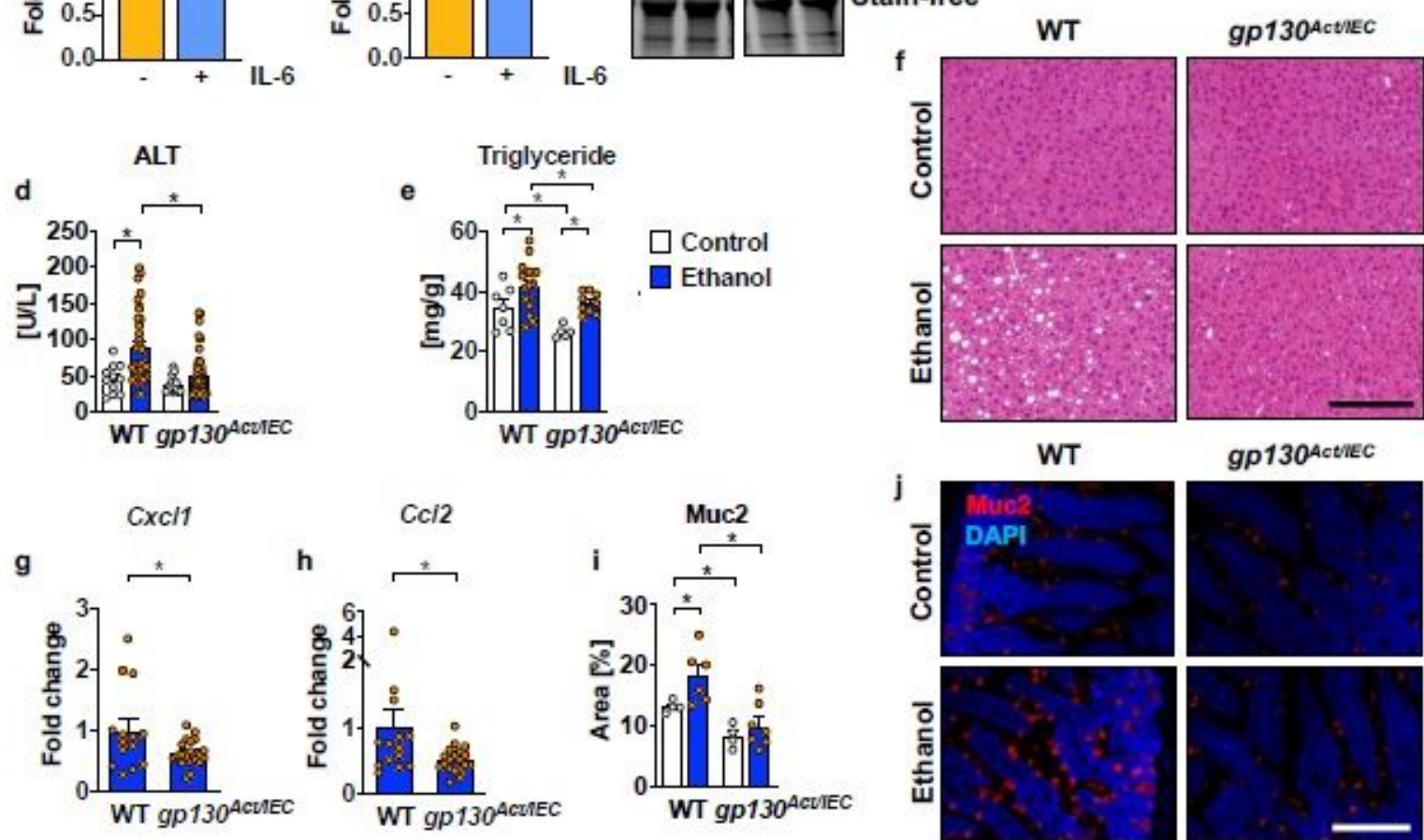

WT
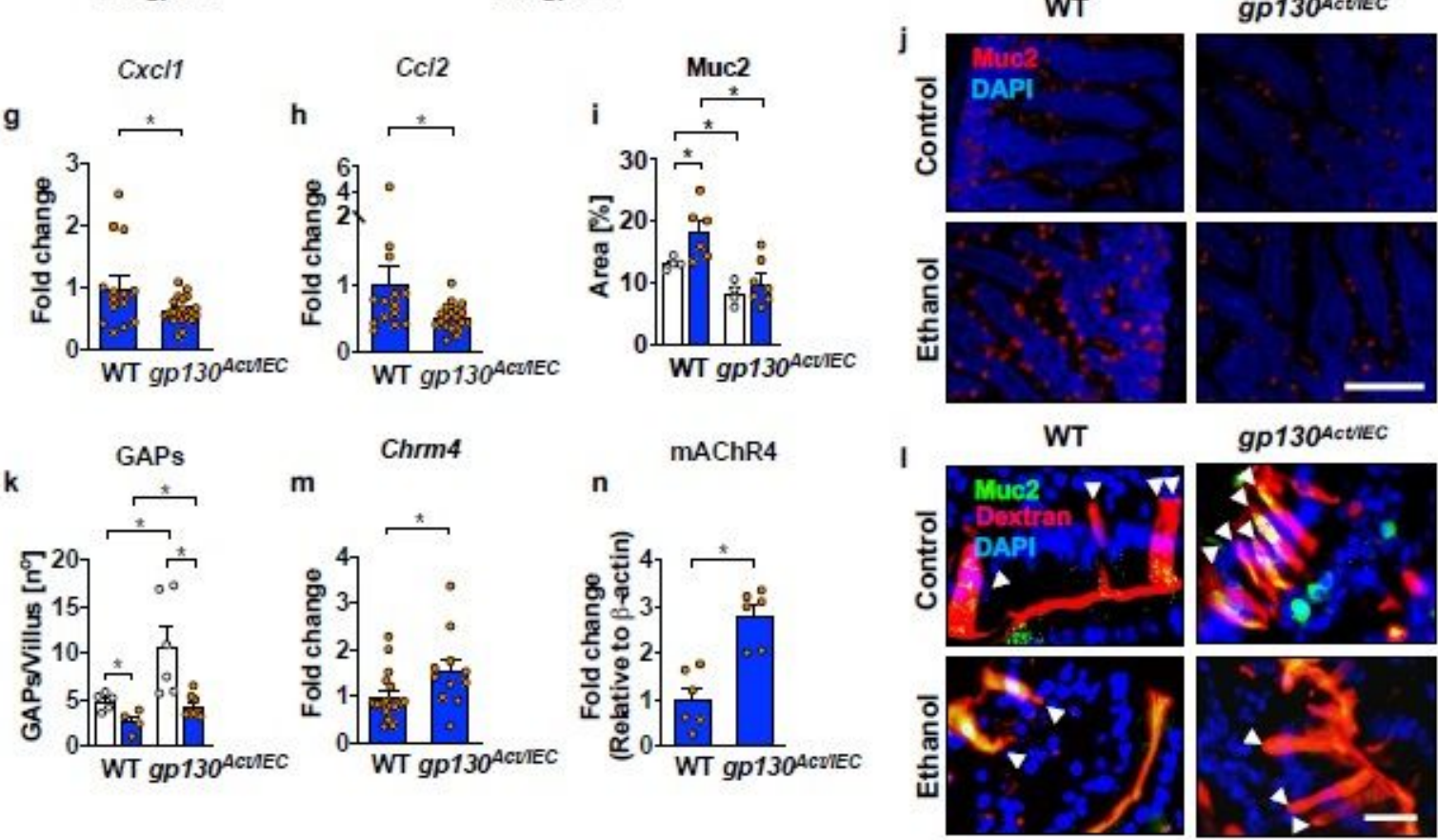

gp130ActiveC

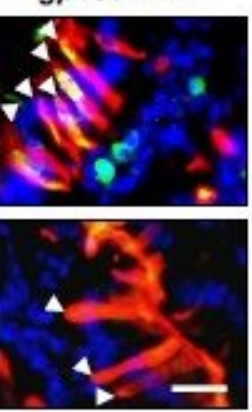

o

Isolated goblet cells

Ethanol

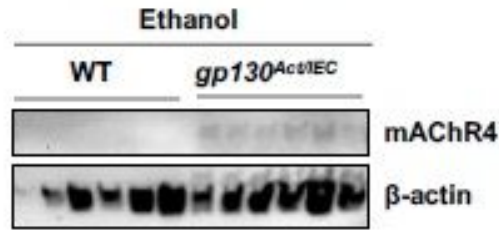

p

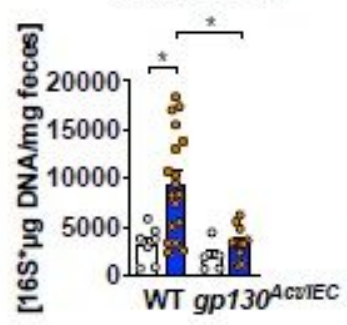

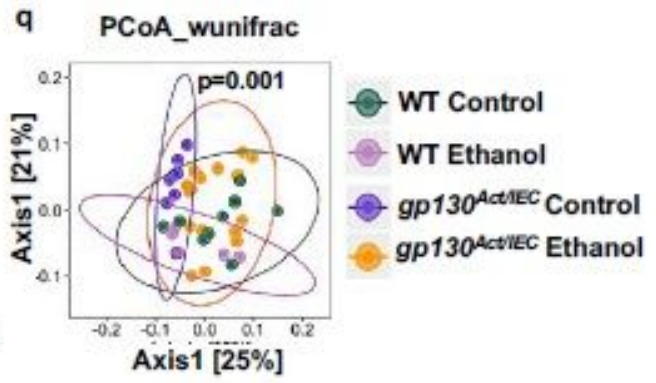

Figure 2

Expression of activated gp130 in IECs prevents ethanol-induced 367 liver disease and promotes GAP formation. (a) Chrm4 mRNA in small intestinal organoids from WT mice $(n=2)$ was quantitated by qPCR after stimulation with IL-6 $(0.02 \mu \mathrm{g} / \mathrm{ml})$ in the presence of ethanol $(10 \mathrm{mM})$ for $12 \mathrm{~h} .6$ technical replicates were pooled in each of two independent biological 371 experiments. (b) mAChR4 protein amounts in WT small intestinal organoids after stimulation without $(n=3)$ or with IL-6 $(0.5 \mu \mathrm{g} / \mathrm{ml})(\mathrm{n}=3)$ for $24 \mathrm{~h}$. (c) 
Representative mAChR4 immunoblots. (d-q) WT mice and gp130Act/IEC littermates were fed control $(n=4-15)$ or ethanol containing Lieber DeCarli diets $(n=4-44)$ for 10 weeks. (d) Plasma levels of ALT. (e) Hepatic triglyceride content. (f) Representative H\&E-stained liver sections. Scale bar $=200 \mu \mathrm{m} .(\mathrm{g}-\mathrm{h})$ Hepatic $\mathrm{Cxcl} 1$ and $\mathrm{Ccl} 2$ mRNAs were quantitated by qPCR. (i) Percentage of Muc2 positive stained area. (j) Representative sections stained with Muc2 antibody (red) showing GCs and DAPI (blue). Scale bar = $200 \mu \mathrm{m}$. (k) Number of GAPs per villus. (I) Representative TMR-dextran (red) showing GAPs, Muc2 antibody (green) and DAPI (blue) stained small intestine sections showing GAPs (Arrowheads). Scale bar $=25 \mu \mathrm{m}$. (m) Small intestinal Chrm4 mRNA was quantified by qPCR. (n) mAChR4 protein amounts relative to b-actin in isolated GCs. (o) Immunoblots of mAChR4 and b-actin in isolated GCs. (p) Total fecal bacteria were quantitated by qPCR. (q) Principal coordinate analysis (PCOA) with weighted UniFrac of 16S rRNA sequencing of fecal samples. $P$ values were determined by One-way ANOVA with Tukey's posthoc test ( $d, e, i, k$ and $p)$, by two sided unpaired Student $t$ test or Mann-Whitney U-statistic test ( $a, b, g, h, m$ and $n$ ) and by two sided paired $t$ test. Results are expressed as mean \pm s.e.m. ${ }^{*} P<0.05$. 

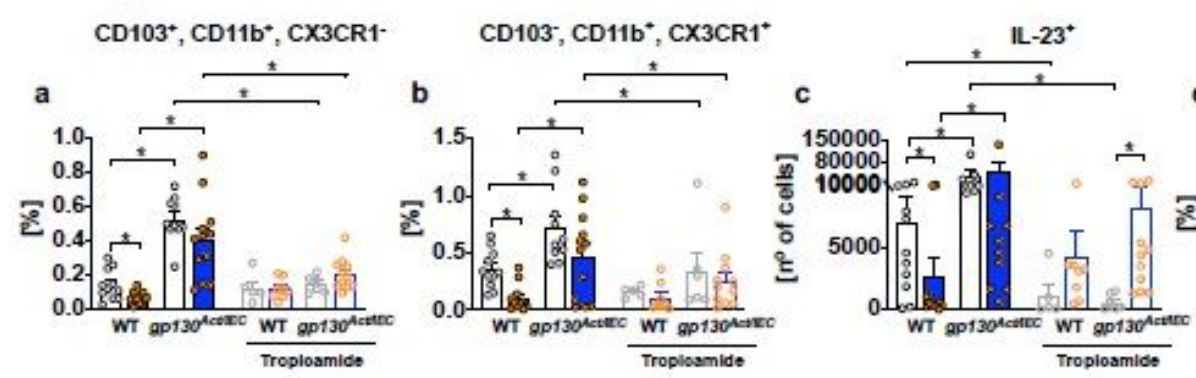

CD3; RORYt+
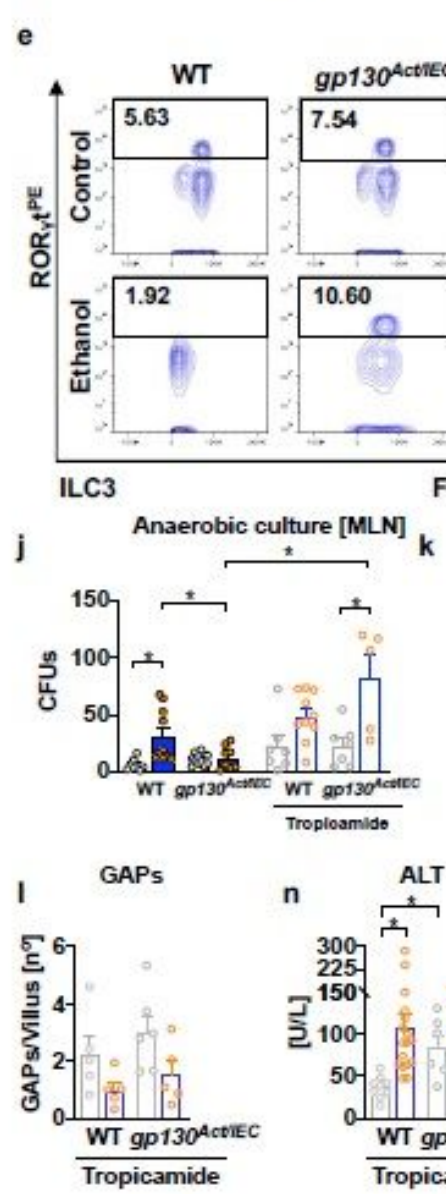

q

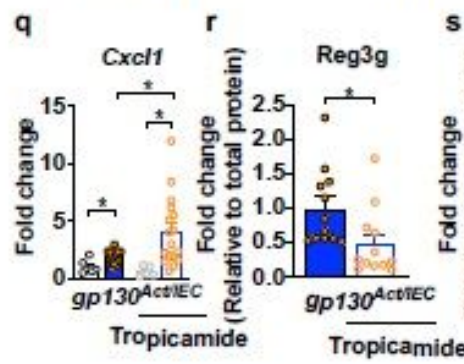

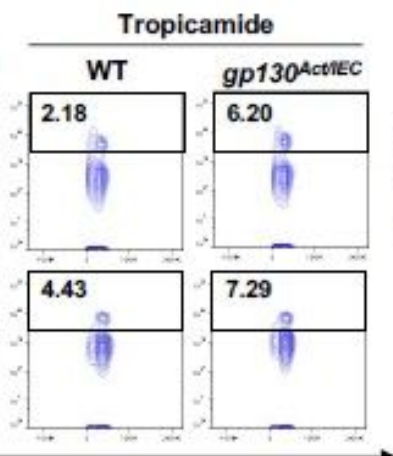

FSC-A

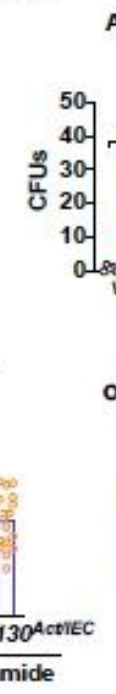

Anaerobic culture [Liver]

f
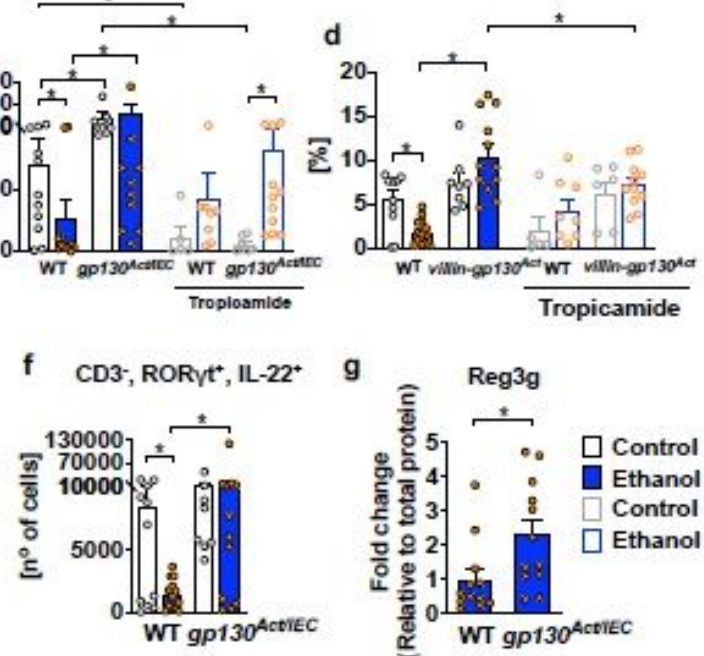

g

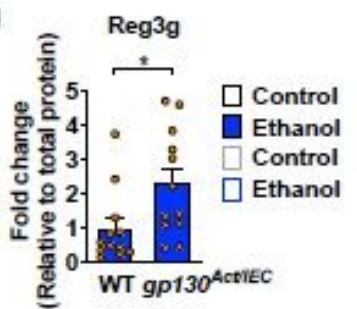

h

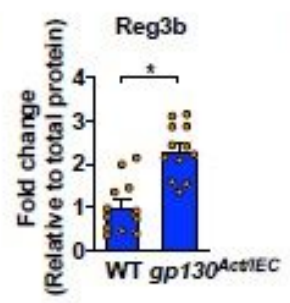

Ethanol

WT gp1304ctiec
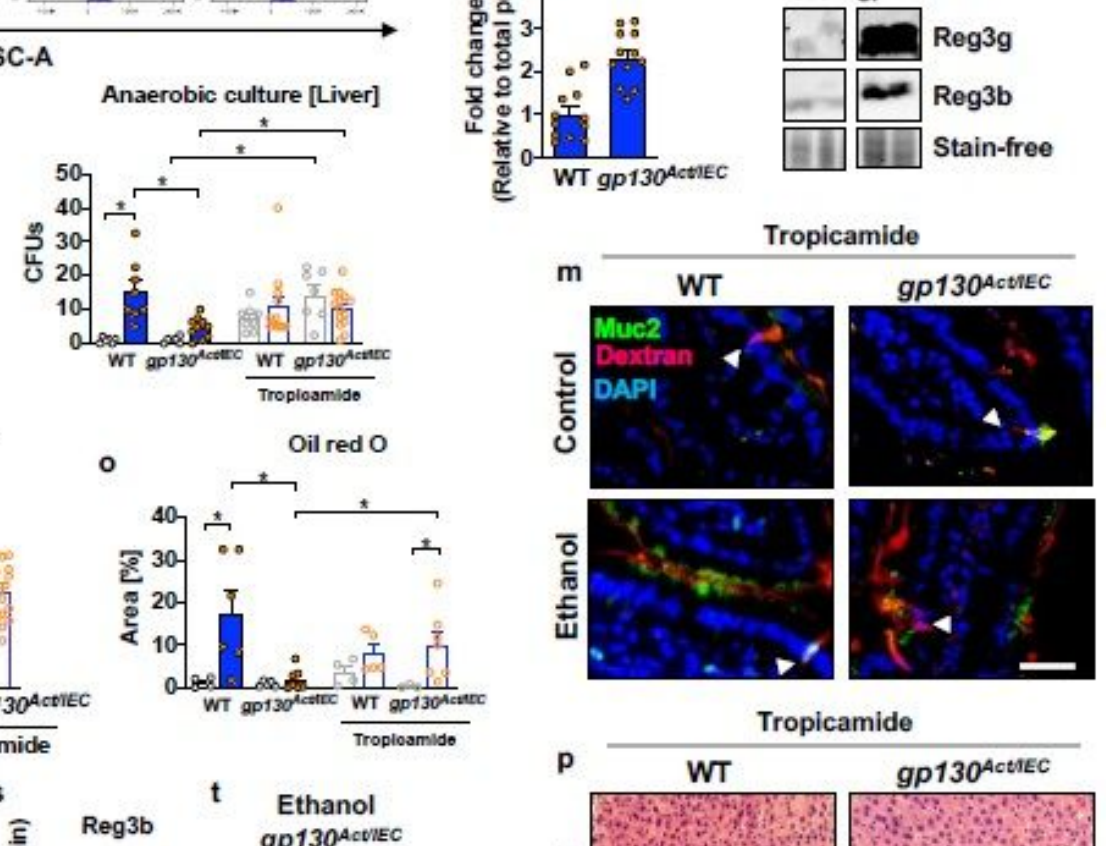

$\mathrm{t}$
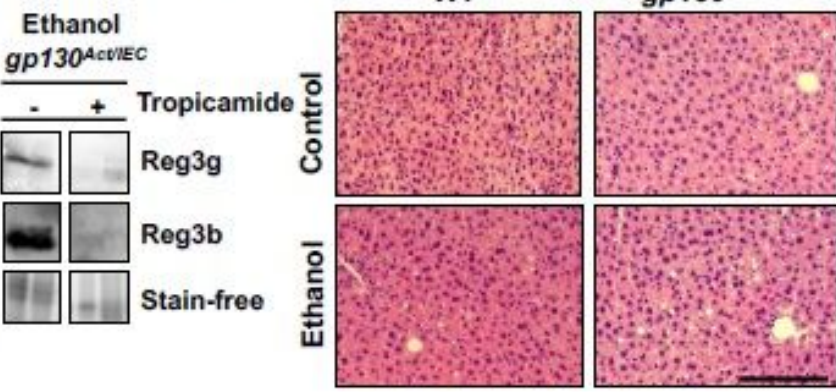

Figure 3

Expression of activated gp130 in IECs stimulates protective 390 intestinal adaptive immune response via mAChR4-mediated GAP formation. ( $a-t)$ WT mice and gp130Act/IEC littermates were fed control $(n=4-$ 11) or ethanol containing Lieber DeCarli diets $(n=6-17)$ for 10 weeks. $(a-d, j-t)$ A group of littermate mice were treated with the mAChR antagonist tropicamide $(20 \mathrm{mg} / \mathrm{kg})$ during the last 29 days $(\mathrm{n}=5-17)$. $(a-c)$ Isolated LP immune cells for APC subset identification studies were stimulated with flagellin (100 
$\mathrm{ng} / \mathrm{ml})$ for $2.5 \mathrm{~h}$. (a-b) Frequencies of tolerogenic APC subsets in total mononuclear phagocyte population were gated according to CD45, MHCII, CD11c, CD103, CD11b, and CX3CR1 expression. (c) Total number of IL-23+ cells in all APCs subsets. (d) Frequencies of ILC3s (CD45+, CD3-, RORDt+) cells after stimulation of isolated LP leukocytes with mouse IL-23 $(40 \mathrm{ng} / \mu \mathrm{l})$ for $4 \mathrm{~h}$. (e) Representative ILC3 and (f) IL-22 expressing ILC3 plots. ( $g-h$ ) Quantification of Reg3g and Reg3b protein amounts relative to total protein identified by stain-free imaging technology. (i) Representative Reg3g and Reg3b immunoblots. (j, k) Number of colony forming units (CFU) of anaerobically cultured bacteria from sterile collected mesenteric lymph nodes and liver. (I) Number of GAPs per villus. (m) Representative TMR-dextran (red), Muc2 antibody (green) and DAPI (blue) stained small intestinal sections showing GAPs (Arrowheads). Scale bar $=25 \mu \mathrm{m}$. (n) Plasma ALT concentrations. (o) Quantification of the Oil red 0 (ORO)-stained liver sections. (p) Representative H\&E stained liver sections. Scale bar $=200 \mu \mathrm{m}$. (q) Hepatic Cxcl1 mRNA quantitated by qPCR. $(r, s)$ Reg3g and Reg3b proteins relative to total protein identified by stain-free imaging technology. ( $t$ ) Representative Reg $3 g$ and Reg3b immunoblots. $P$ values were determined by two way ( $a-d, j-k$ and $o$ ) or one-way ANOVA ( $f, l, n$ and $q$ ) with Tukey's post-hoc test and by two sided unpaired Student $t$ test or Mann-Whitney U-statistic test ( $g, h, r$ and $s$ ). Results are expressed as mean \pm s.e.m. ${ }^{*} \mathrm{P}<0.05$. 


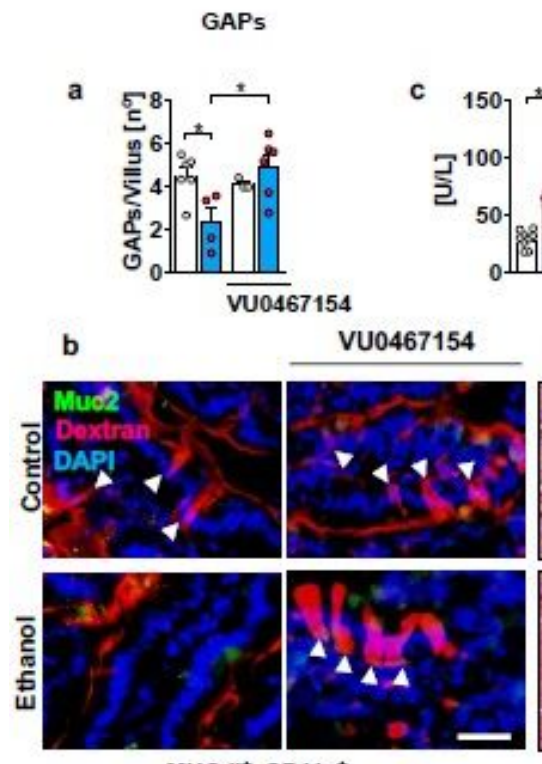

MHC-II+, CD11c ${ }^{+}$

h

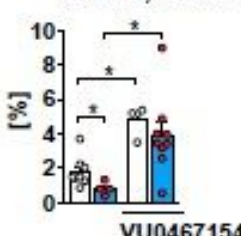

j
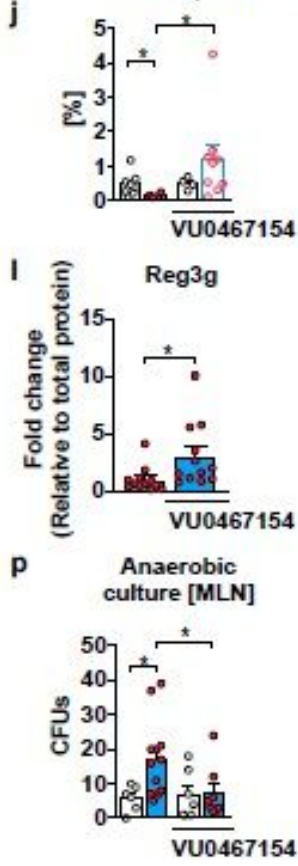

$\mathbf{i}$
$\frac{0}{0}$
$\frac{5}{0}$

m
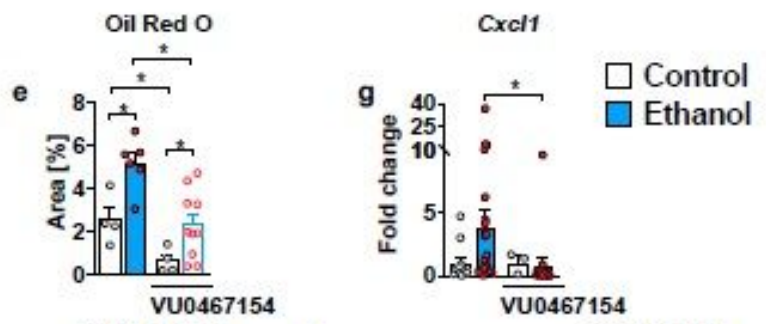

vu0467154

d

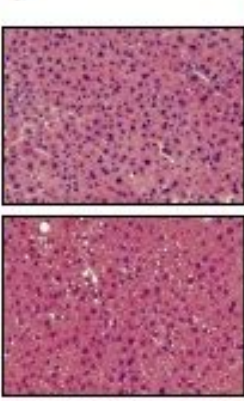

VU0467154
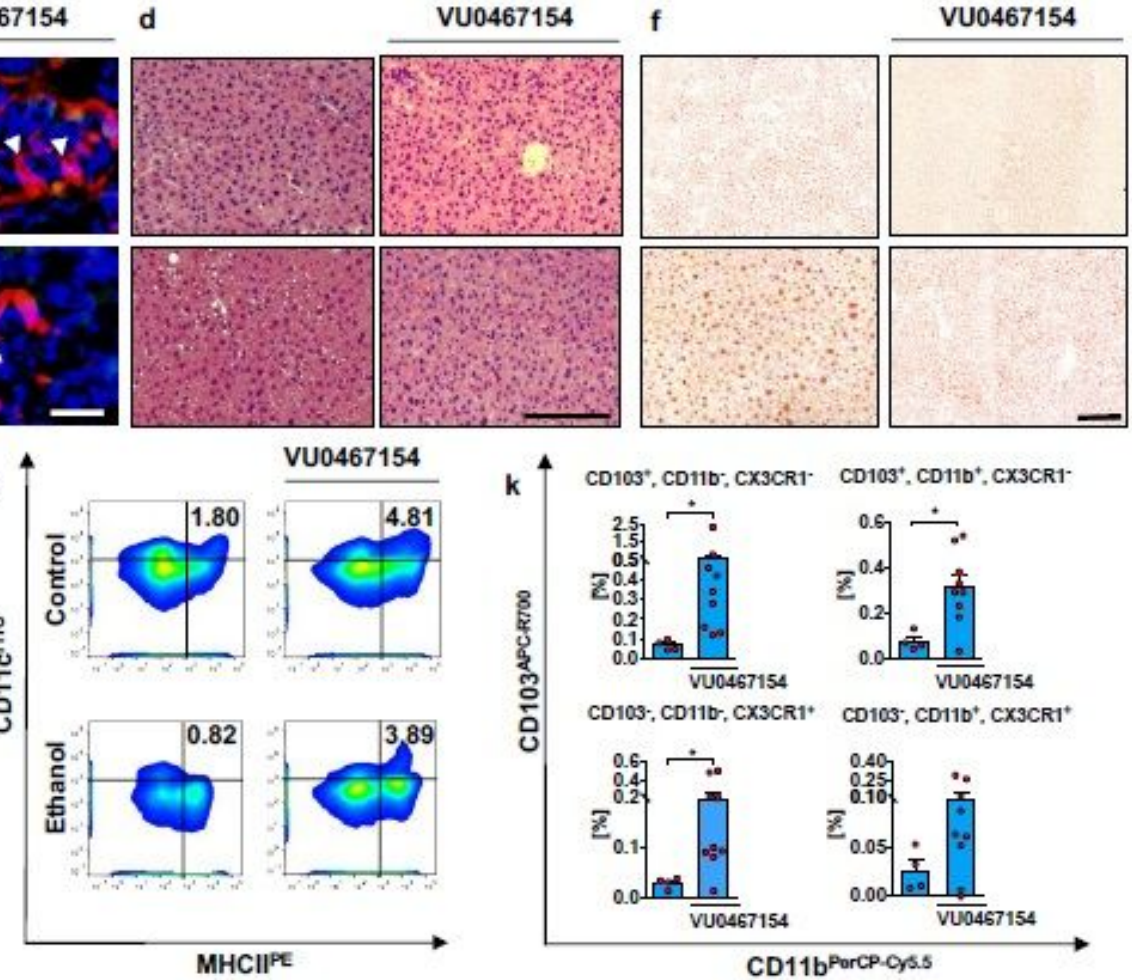

CD103; CD116, CX3CR1 ${ }^{*}$ CD103; CD116 $^{+}, \mathrm{CX}^{2} \mathrm{CRI}^{+}$

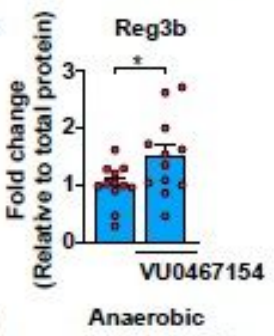

$n$ อ $\mathrm{IL}-10$

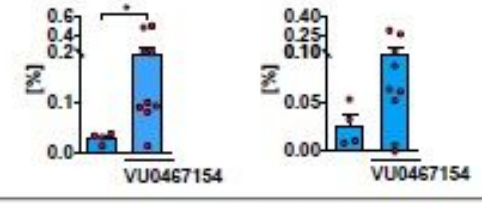

CD11b PorCP-Cys.5

Ethano

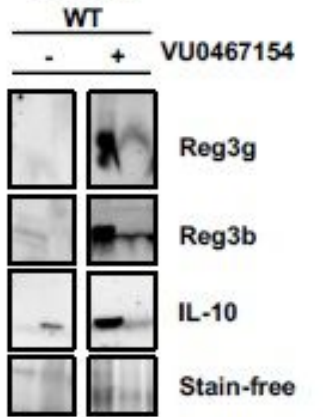

\section{Figure 4}

A mAChR4 PAM reduces ethanol-induced liver disease. 413 WT mice were fed control $(n=3-9)$ or ethanol containing Lieber DeCarli diets $(n=4-38)$ for 10 weeks in the presence or absence of the specific mAChR4 PAM VU0467154 (5 mg/Kg), dissolved in the diet during the last 29 days. (a) Number of GAPs per villus. (b) Representative TMR-dextran (red), Muc2 (green) and DAPI (blue) stained small intestine sections showing GAPs (Arrowheads). Scale bar $=25 \mu \mathrm{m}$. (c) Plasma ALT concentrations. (d) Representative H\&E 
stained liver sections. Scale bar $=200 \mu \mathrm{m}$. (e) Quantification of ORO-stained liver sections. (f) Representative oil ORO-stained liver sections. Scale bar $=100 \mu \mathrm{m}$. (g) Hepatic Cxcl1 mRNA amounts. (hk) DCs were stimulated with flagellin $(100 \mathrm{ng} / \mathrm{ml})$ for $2.5 \mathrm{~h}$ before staining. (h) Frequencies of CD45+, $\mathrm{MHCll}+\mathrm{CD} 11 \mathrm{C}+\mathrm{APCs}$ in total mononuclear phagocyte population. (i) Representative APCs plots. (j) Frequencies of CD45+, MHCll+, CD11ct, CD103+, CD11b+ DCs. (k) Frequencies of tolerogenic APC subsets according to CD45, MHCII, CD11C, CD103, CD11b and CX3CR1 expression. (I-n) Quantification of Reg3g, Reg3b and IL-10 proteins relative to total protein identified by stain-free imaging technology. (o) Representative Reg3g, Reg3b, and IL-10 immunoblots. $(p, q)$ Number of CFUs of anaerobically cultured bacteria from sterile collected MLNs and liver. P values were determined by one-way ANOVA (a, c, e, g, h, j, $\mathrm{p}, \mathrm{q})$ with Tukey's post-hoc test and by two-sided unpaired Student $t$ test or Mann-Whitney U-statistic test $(k, I-n)$. Results are expressed as mean \pm s.e.m. ${ }^{*} \mathrm{P}<0.05$.

\section{Supplementary Files}

This is a list of supplementary files associated with this preprint. Click to download.

- ExtDataFigGAPsNatureV5.pptx.pdf 